\title{
Negative Hyperconjugation in phosphorus stabilized the
}

\section{carbanions}

\author{
Tom Leyssens, * Daniel Peeters
}

Laboratoire de Chimie Quantique, Université Catholique de Louvain, Place Louis Pasteur 1, B-1348

Louvain-la-Neuve (Belgium). E-mail : t.leyssens@chim.ucl.ac.be

\section{Supporting Information}

This file contains optimized Z-matrix coordinates in $\AA$ at the B3LYP and MP2 level for all the species discussed in the text.

S2: z-matrices of all studied compounds at B3LYP and MP2 level of theory. 


$\begin{array}{lc}\text { HCP2 } & 142.3036 \\ \text { HCP3 } & 89.3447 \\ \text { DI1 } & 98.7092 \\ \text { DI2 } & 198.4502 \\ \text { DI3 } & 130.4727 \\ \text { DI4 } & -108.8041\end{array}$

$\mathrm{CH}_{2} \mathrm{PH}_{2}^{-}$

$B 3 L Y P$

$\mathrm{P}$

$\mathrm{H} 1 \mathrm{PH}$

H 1 PH2 2 HPH

C 1 PC 2 PCH 3 DI1

$\mathrm{H} 4 \mathrm{CH} 2 \mathrm{HCP} 3$ DI2

$\mathrm{H} 4 \mathrm{CH} 22 \mathrm{HCP} 25$ DI3

$\begin{array}{lc}\text { PH } & 1.445 \\ \text { PH2 } & 1.4876 \\ \text { PC } & 1.7612 \\ \text { CH } & 1.0914 \\ \text { CH2 } & 1.0934 \\ \text { HPH } & 89.1136 \\ \text { PCH } & 104.8971 \\ \text { HCP } & 143.3746 \\ \text { HCP2 } & 101.4992 \\ \text { DI1 } & 113.3218 \\ \text { DI2 } & 281.4026 \\ \text { DI3 } & 166.7692\end{array}$

$M P 2$

$-11$

$\mathrm{P}$

$\mathrm{H} 1 \mathrm{PH}$

$\mathrm{H} 1 \mathrm{PH} 22 \mathrm{HPH}$

C 1 PC 2 PCH 3 DI 1

$\mathrm{H} 4 \mathrm{CH} 2 \mathrm{HCP} 3$ DI2

$\mathrm{H} 4 \mathrm{CH} 22 \mathrm{HCP} 25 \mathrm{DI} 3$

$\mathrm{X} 4 \mathrm{CH} 32 \mathrm{HCP} 35$ DI4

$\begin{array}{lc}\text { PH } & 1.4234 \\ \text { PH2 } & 1.4549 \\ \text { PC } & 1.7593 \\ \text { CH } & 1.0867 \\ \text { CH2 } & 1.0892 \\ \text { CH3 } & 1.0441 \\ \text { HPH } & 90.5442 \\ \text { PCH } & 105.0279 \\ \text { HCP } & 142.5359 \\ \text { HCP2 } & 102.0417 \\ \text { HCP3 } & 88.4457 \\ \text { DI1 } & 113.6816 \\ \text { DI2 } & 281.2468 \\ \text { DI3 } & 165.0424 \\ \text { DI4 } & -94.5555\end{array}$

$B 3 L Y P / E(D E L)$

$\mathrm{P}$

$\mathrm{H} 1 \mathrm{PH}$

$\mathrm{H} 1 \mathrm{PH} 22 \mathrm{HPH}$

C 1 PC 2 PCH 3 DI 1

$\mathrm{H} 4 \mathrm{CH} 2 \mathrm{HCP} 3$ DI 2

H 4 CH3 2 HCP3 5 DI4

$\begin{array}{lc}\text { PH } & 1.4306 \\ \text { PH2 } & 1.4391 \\ \text { PC } & 1.9354 \\ \text { CH } & 1.1185 \\ \text { CH3 } & 1.1124 \\ \text { HPH } & 92.844 \\ \text { PCH } & 96.0533 \\ \text { HCP } & 88.1275 \\ \text { HCP3 } & 78.497 \\ \text { DI1 } & 103.6442 \\ \text { DI2 } & 202.1442 \\ \text { DI4 } & -104.9965\end{array}$

$\mathrm{C}_{2} \mathrm{H}_{4} \mathrm{PH}_{2}^{-}$

$B 3 L Y P$

$\mathrm{C}$

$\mathrm{C} 1 \mathrm{CC}$

$\mathrm{H} 1 \mathrm{CH} 2 \mathrm{CHH} 1$

$\mathrm{H} 1 \mathrm{CH} 22 \mathrm{CHH} 23 \mathrm{di} 1$

$\mathrm{H} 1 \mathrm{CH} 32 \mathrm{CHH} 34$ di2

$\mathrm{X} 2 \mathrm{CH} 41 \mathrm{CHH} 43$ di 3

H 2 CH5 1 CHH5 6 di4

P 2 CP 1 PCC 6 di 5

H 8 PH 2 HPC 6 di 6

H 8 PH2 2 HPC2 9 di7

CC $\quad 1.5115$

$\mathrm{CH} \quad 1.1213$

$\mathrm{CH} 2 \quad 1.1034$

$\mathrm{CH} 3 \quad 1.1034$

CH4 1.0964

CH5 1.0944

CP 1.76

$\mathrm{PH} \quad 1.5011$

PH2 1.4448

CHH1 115.2289

CHH2 111.8454

CHH3 111.5337

CHH4 110.6786

CHH5 115.0337

PCC 120.9696

HPC 109.6392

HPC2 102.9938

di1 121.555

di2 119.0508

di3 181.5803

di4 97.2146

di5 -123.5922

di6 194.887

di7 93.526

$M P 2$

$\mathrm{C}$

$\mathrm{C} 1 \mathrm{CC}$

$\mathrm{H} 1 \mathrm{CH} 2 \mathrm{CHH} 1$

$\mathrm{H} 1 \mathrm{CH} 22 \mathrm{CHH} 23$ di 1

$\mathrm{H} 1 \mathrm{CH} 32 \mathrm{CHH} 34 \mathrm{di} 2$

$\mathrm{X} 2 \mathrm{CH} 41 \mathrm{CHH} 43$ di 3

H 2 CH5 1 CHH5 6 di4

P 2 CP 1 PCC 6 di 5

H 8 PH 2 HPC 6 di 6

H 8 PH2 2 HPC2 9 di7

$\begin{array}{lc}\mathrm{CC} & 1.5099 \\ \mathrm{CH} & 1.1131 \\ \mathrm{CH} 2 & 1.0974 \\ \mathrm{CH} 3 & 1.0971 \\ \mathrm{CH} 4 & 1.1283 \mathrm{~F} \\ \mathrm{CH} 5 & 1.0903 \\ \mathrm{CP} & 1.7583 \\ \mathrm{PH} & 1.4716 \\ \mathrm{PH} 2 & 1.4242 \\ \mathrm{CHH} 1 & 114.7531 \\ \mathrm{CHH} 2 & 111.8095 \\ \mathrm{CHH} 3 & 111.0832 \\ \mathrm{CHH} 4 & 127.7332 \mathrm{~F} \\ \mathrm{CHH} 5 & 114.7112 \\ \mathrm{PCC} & 118.4722 \\ \mathrm{HPC} & 108.6561 \\ \mathrm{HPC} 2 & 102.557 \\ \text { di1 } & 121.795 \\ \text { di2 } & 119.0199 \\ \text { di3 } & 178.5833 \mathrm{~F} \\ \text { di4 } & 102.3956 \\ \text { di5 } & -121.7677 \\ \text { di6 } & 184.6473 \\ \text { di7 } & 94.3536\end{array}$

$\mathrm{C}_{3} \mathrm{H}_{4} \mathrm{PH}_{2}^{-}$

$B 3 L Y P$

$\mathrm{C}$

C 1 CC

C 2 CC 11 CCC

H 1 CH1 2 HCC1 3 DI 1

$\mathrm{H} 1 \mathrm{CH} 22 \mathrm{HCC} 24 \mathrm{DI} 2$

$\mathrm{H} 2 \mathrm{CH} 31 \mathrm{HCC} 34 \mathrm{DI} 3$

$\mathrm{H} 3 \mathrm{CH} 42 \mathrm{HCC} 46$ DI4

P 3 CH5 2 HCC5 7 DI5

H 8 PH1 3 HPC1 2 DI6

H 8 PH2 3 HPC2 9 DI7

$\begin{array}{ll}\text { CC } & 1.3835 \\ \text { CC1 } & 1.4134 \\ \text { CH1 } & 1.0895 \\ \text { CH2 } & 1.087 \\ \text { CH3 } & 1.0971 \\ \text { CH4 } & 1.0914 \\ \text { CH5 } & 1.7705 \\ \text { PH1 } & 1.4523 \\ \text { PH2 } & 1.4524 \\ \text { CCC } & 130.3709 \\ \text { HCC1 } & 121.9265 \\ \text { HCC2 } & 120.9858 \\ \text { HCC3 } & 115.1158 \\ \text { HCC4 } & 118.4008 \\ \text { HCC5 } & 120.7306 \\ \text { HPC1 } & 106.197 \\ \text { HPC2 } & 106.2103 \\ \text { DI1 } & -0.0288 \\ \text { DI2 } & 180.0412 \\ \text { DI3 } & 179.977 \\ \text { DI4 } & 180.0254 \\ \text { DI5 } & 179.9387 \\ \text { DI6 } & 132.6393 \\ \text { DI7 } & 94.8455\end{array}$

$M P 2$

$\mathrm{C}$

C 1 CC

C 2 CC $11 \mathrm{CCC}$

H 1 CH1 2 HCC1 3 DI 1

$\mathrm{H} 1 \mathrm{CH} 22 \mathrm{HCC} 24$ DI2

$\mathrm{H} 2 \mathrm{CH} 31$ HCC3 4 DI3

$\mathrm{H} 3 \mathrm{CH} 42 \mathrm{HCC} 46$ DI4

P 3 CH5 2 HCC5 7 DI5

H 8 PH1 3 HPC1 2 DI6

H 8 PH2 3 HPC2 9 DI7

CC 1.3832

CC1 1.4128

CH1 1.0852

$\mathrm{CH} 2 \quad 1.0828$

CH3 1.0938

CH4 1.0877

CH5 1.7641

PH1 1.4301

PH2 1.4301

CCC 130.1886

HCC1 121.8088

$\begin{array}{ll}\mathrm{HCC} 2 & 120.8188\end{array}$

HCC3 115.1785

HCC4 118.6416

HCC5 119.8888

HPC1 106.3011

HPC2 106.3094

DI1 $\quad-0.0299$

DI2 180.0318

DI3 179.9853

DI4 180.0066

DI5 179.9499

DI6 131.8162

DI7 96.431 


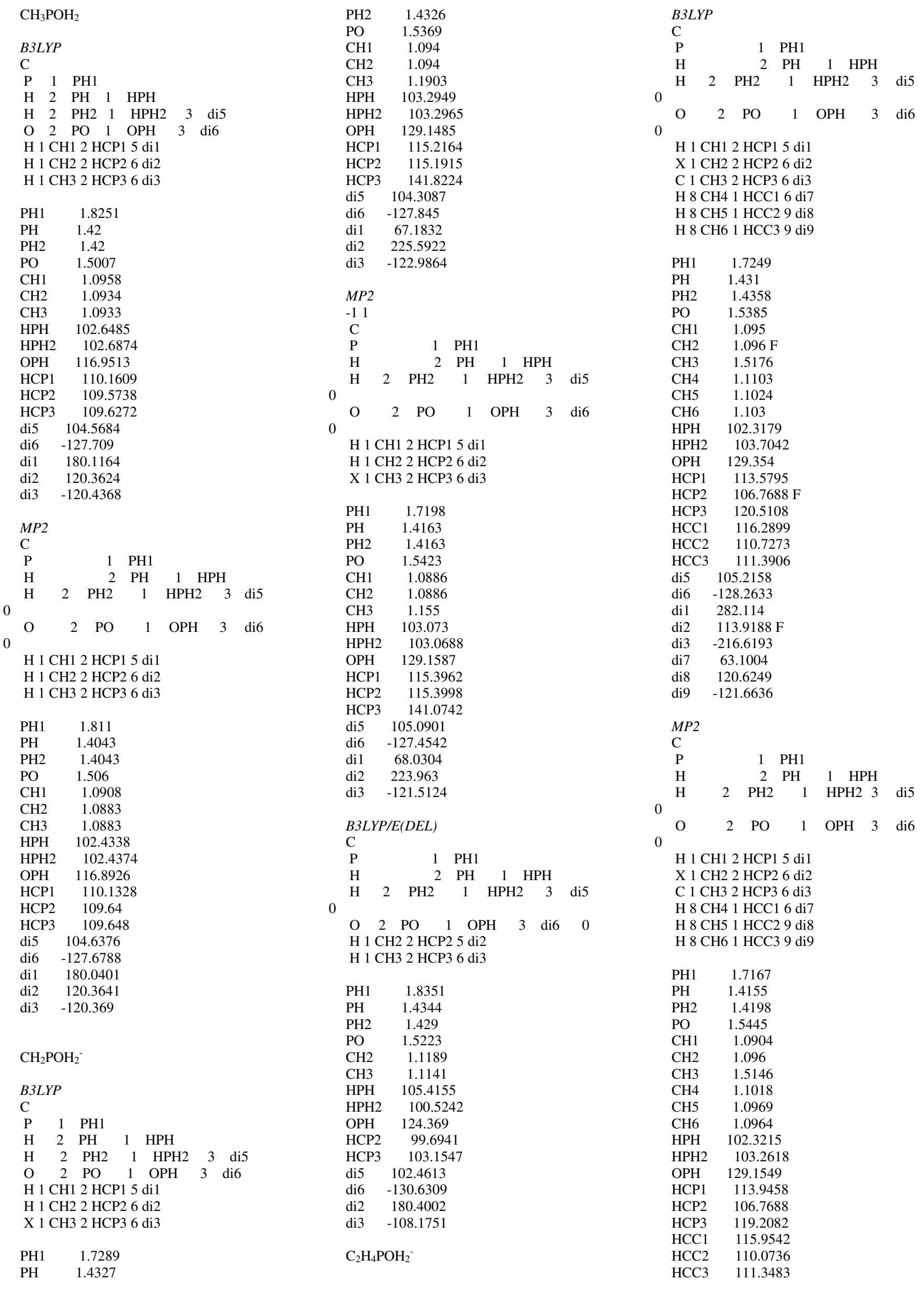




$\begin{array}{lc}\text { di5 } & 105.8678 \\ \text { di6 } & -128.2703 \\ \text { di1 } & 282.3081 \\ \text { di2 } & 113.9188 \\ \text { di3 } & -217.4837 \\ \text { di7 } & 61.4802 \\ \text { di8 } & 120.344 \\ \text { di9 } & -122.0038\end{array}$

$\mathrm{C}_{3} \mathrm{H}_{4} \mathrm{POH}_{2}{ }^{-}$

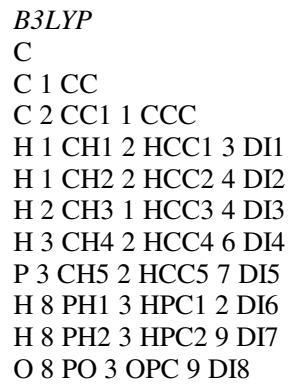

$\begin{array}{lc}\text { CC } & 1.373 \\ \text { CC1 } & 1.4222 \\ \text { CH1 } & 1.0889 \\ \text { CH2 } & 1.0867 \\ \text { CH3 } & 1.0967 \\ \text { CH4 } & 1.0909 \\ \text { CH5 } & 1.7338 \\ \text { PH1 } & 1.4305 \\ \text { PH2 } & 1.4303 \\ \text { PO } & 1.5276 \\ \text { CCC } & 129.9938 \\ \text { HCC1 } & 121.8048 \\ \text { HCC2 } & 121.0831 \\ \text { HCC3 } & 115.2293 \\ \text { HCC4 } & 119.143 \\ \text { HCC5 } & 122.8594 \\ \text { HPC1 } & 104.8902 \\ \text { HPC2 } & 102.4233 \\ \text { OPC } & 126.2367 \\ \text { DI1 } & 2.5196 \\ \text { DI2 } & 180.3217 \\ \text { DI3 } & 180.8334 \\ \text { DI4 } & 177.2044 \\ \text { DI5 } & 192.3396 \\ \text { DI6 } & 219.4102 \\ \text { DI7 } & 105.3691 \\ \text { DI8 } & -126.7799 \\ & \end{array}$

$M P 2$

$\mathrm{C}$

C 1 CC

C 2 CC $11 \mathrm{CCC}$

H 1 CH1 2 HCC1 3 DI 1

$\mathrm{H} 1 \mathrm{CH} 22 \mathrm{HCC} 24 \mathrm{DI} 2$

$\mathrm{H} 2 \mathrm{CH} 31 \mathrm{HCC} 34 \mathrm{DI} 3$

H 3 CH4 2 HCC4 6 DI4

P 3 CH5 2 HCC5 7 DI5

H 8 PH1 3 HPC1 2 DI6

H 8 PH2 3 HPC2 9 DI7

O 8 PO 3 OPC 9 DI8

$\begin{array}{lc}\mathrm{CC} & 1.3711 \\ \mathrm{CC} 1 & 1.4258 \\ \mathrm{CH} 1 & 1.0844 \\ \mathrm{CH} 2 & 1.0824 \\ \mathrm{CH} 3 & 1.0934 \\ \mathrm{CH} 4 & 1.0874 \\ \mathrm{CH} 5 & 1.7262 \\ \mathrm{PH} 1 & 1.4148 \\ \mathrm{PH} 2 & 1.4149 \\ \mathrm{PO} & 1.5341 \\ \mathrm{CCC} & 129.4909 \\ \mathrm{HCC} 1 & 121.5442\end{array}$

$\begin{array}{ll}\text { HCC2 } & 121.0006 \\ \text { HCC3 } & 115.4517 \\ \text { HCC4 } & 118.6697 \\ \text { HCC5 } & 120.8562 \\ \text { HPC1 } & 104.3573 \\ \text { HPC2 } & 101.6695 \\ \text { OPC } & 126.8113 \\ \text { DI1 } & 4.0775 \\ \text { DI2 } & 179.7984 \\ \text { DI3 } & 181.0331 \\ \text { DI4 } & 175.551 \\ \text { DI5 } & 200.3827 \\ \text { DI6 } & 213.2175 \\ \text { DI7 } & 105.618 \\ \text { DI8 } & -127.2489\end{array}$

$\mathrm{CH}_{3} \mathrm{PHCH}_{3}$

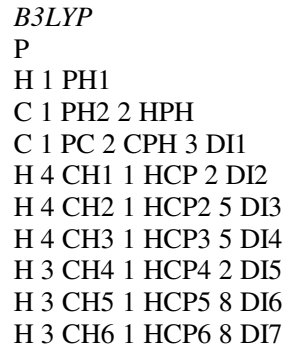

$\begin{array}{lc}\text { PH1 } & 1.4258 \\ \text { PH2 } & 1.871 \\ \text { PC } & 1.871 \\ \text { CH1 } & 1.0944 \\ \text { CH2 } & 1.0946 \\ \text { CH3 } & 1.0948 \\ \text { CH4 } & 1.0944 \\ \text { CH5 } & 1.0947 \\ \text { CH6 } & 1.0946 \\ \text { HPH } & 96.9245 \\ \text { CPH } & 96.8988 \\ \text { HCP } & 109.2612 \\ \text { HCP2 } & 109.1392 \\ \text { HCP3 } & 113.1878 \\ \text { HCP4 } & 109.2809 \\ \text { HCP5 } & 113.206 \\ \text { HCP6 } & 109.0522 \\ \text { DI1 } & 101.291 \\ \text { DI2 } & 70.2462 \\ \text { DI3 } & 117.3067 \\ \text { DI4 } & -121.466 \\ \text { DI5 } & -70.2523 \\ \text { DI6 } & 121.5288 \\ \text { DI7 } & -117.2861 \\ & \end{array}$

$M P 2$

$\mathrm{P}$

H 1 PH1

C $1 \mathrm{PH} 22 \mathrm{HPH}$

C 1 PC 2 CPH 3 DI 1

$\mathrm{H} 4 \mathrm{CH} 11$ HCP 2 DI2

$\mathrm{H} 4 \mathrm{CH} 21 \mathrm{HCP} 25 \mathrm{DI} 3$

H 4 CH3 1 HCP3 5 DI4

H 3 CH4 1 HCP4 2 DI5

H 3 CH5 1 HCP5 8 DI6

H 3 CH6 1 HCP6 8 DI7

$\begin{array}{ll}\text { PH1 } & 1.4096 \\ \text { PH2 } & 1.8537 \\ \text { PC } & 1.8537 \\ \mathrm{CH} 1 & 1.0895 \\ \mathrm{CH} 2 & 1.0897 \\ \mathrm{CH} 3 & 1.09 \\ \mathrm{CH} 4 & 1.0895 \\ \mathrm{CH} 5 & 1.09 \\ \mathrm{CH} 6 & 1.0897 \\ \mathrm{HPH} & 97.3956\end{array}$

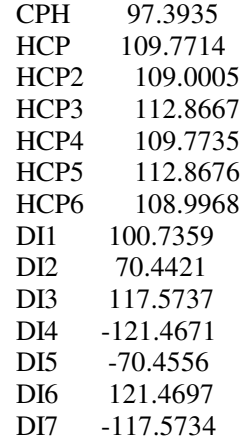

$\mathrm{CH}_{2} \mathrm{PHCH}_{3}{ }^{-}$

B3LYP

$\mathrm{P}$

H 1 PH1

C $1 \mathrm{PH} 22 \mathrm{HPH}$

C 1 PC 2 CPH 3 DI1

H 4 CH1 1 HCP 2 DI2

$\mathrm{H} 4 \mathrm{CH} 21 \mathrm{HCP} 25 \mathrm{DI} 3$

H 4 CH3 1 HCP3 5 DI4

H 3 CH4 1 HCP4 2 DI5

H 3 CH5 1 HCP5 8 DI6

X 3 CH6 1 HCP6 8 DI7

$\begin{array}{lc}\text { PH1 } & 1.4613 \\ \text { PH2 } & 1.7433 \\ \text { PC } & 1.9162 \\ \text { CH1 } & 1.1035 \\ \text { CH2 } & 1.0971 \\ \text { CH3 } & 1.0987 \\ \text { CH4 } & 1.0867 \\ \text { CH5 } & 1.091 \\ \text { CH6 } & 1.0918 \\ \text { HPH } & 106.7038 \\ \text { CPH } & 90.4598 \\ \text { HCP } & 109.9974 \\ \text { HCP2 } & 108.911 \\ \text { HCP3 } & 111.7272 \\ \text { HCP4 } & 117.6761 \\ \text { HCP5 } & 124.8422 \\ \text { HCP6 } & 103.9553 \\ \text { DI1 } & 109.3141 \\ \text { DI2 } & 56.6531 \\ \text { DI3 } & 119.3879 \\ \text { DI4 } & -121.1781 \\ \text { DI5 } & -134.9813 \\ \text { DI6 } & 171.0721 \\ \text { DI7 } & -113.5456 \\ & \end{array}$

MP2

$\mathrm{P}$

H 1 PH1

C 1 PH2 $2 \mathrm{HPH}$

C 1 PC 2 CPH 3 DI1

$\mathrm{H} 4 \mathrm{CH} 11 \mathrm{HCP} 2 \mathrm{DI} 2$

$\mathrm{H} 4 \mathrm{CH} 21 \mathrm{HCP} 25 \mathrm{DI} 3$

$\mathrm{H} 4 \mathrm{CH} 31 \mathrm{HCP} 35 \mathrm{DI} 4$

H 3 CH4 1 HCP4 2 DI5

H 3 CH5 1 HCP5 8 DI6

$\mathrm{X} 3 \mathrm{CH} 61 \mathrm{HCP} 68$ DI7

$\begin{array}{lc}\text { PH1 } & 1.4459 \\ \text { PH2 } & 1.7465 \\ \text { PC } & 1.883 \\ \text { CH1 } & 1.0976 \\ \text { CH2 } & 1.0911 \\ \text { CH3 } & 1.0933 \\ \text { CH4 } & 1.0844 \\ \text { CH5 } & 1.0884 \\ \text { CH6 } & 1.0918 \\ \text { HPH } & 108.623\end{array}$




$\begin{array}{lc}\text { CPH } & 90.7685 \\ \text { HCP } & 110.3498 \\ \text { HCP2 } & 109.0176 \\ \text { HCP3 } & 111.2951 \\ \text { HCP4 } & 116.0708 \\ \text { HCP5 } & 122.4613 \\ \text { HCP6 } & 103.9553 \\ \text { DI1 } & 106.8644 \\ \text { DI2 } & 55.5548 \\ \text { DI3 } & 119.7552 \\ \text { DI4 } & -121.0273 \\ \text { DI5 } & -122.867 \\ \text { DI6 } & 151.855 \\ \text { DI7 } & -113.5456\end{array}$

$\mathrm{C}_{2} \mathrm{H}_{4} \mathrm{PHCH}_{3}{ }^{-}$

B3LYP

$\mathrm{P}$

H 1 PH1

C 1 PH2 $2 \mathrm{HPH}$

C 1 PC 2 CPH 3 DI 1

H 4 CH1 1 HCP 2 DI2

$\mathrm{H} 4 \mathrm{CH} 21$ HCP2 5 DI 3

$\mathrm{H} 4 \mathrm{CH} 31 \mathrm{HCP} 35 \mathrm{DI} 4$

$\mathrm{X} 3 \mathrm{CH} 41 \mathrm{HCP} 42$ DI5

H 3 CH5 1 HCP5 8 DI6

C 3 CH6 1 HCP6 8 DI7

H 10 CH7 3 HCC1 8 DI8

H 10 CH8 3 HCC2 11 DI9

H 10 CH9 3 HCC3 11 DI10

$\begin{array}{lc}\text { PH1 } & 1.4859 \\ \text { PH2 } & 1.7527 \\ \text { PC } & 1.9028 \\ \text { CH1 } & 1.1008 \\ \text { CH2 } & 1.0965 \\ \text { CH3 } & 1.0978 \\ \text { CH4 } & 1.0968 \mathrm{~F} \\ \text { CH5 } & 1.0919 \\ \text { CH6 } & 1.5134 \\ \text { CH7 } & 1.1024 \\ \text { CH8 } & 1.1195 \\ \text { CH9 } & 1.1014 \\ \text { HPH } & 107.1272 \\ \text { CPH } & 90.4867 \\ \text { HCP } & 109.3659 \\ \text { HCP2 } & 108.9254 \\ \text { HCP3 } & 112.7259 \\ \text { HCP4 } & 110.3484 \text { F } \\ \text { HCP5 } & 112.7487 \\ \text { HCP6 } & 122.7633 \\ \text { HCC1 } & 111.548 \\ \text { HCC2 } & 114.6727 \\ \text { HCC3 } & 112.6861 \\ \text { DI1 } & 107.0106 \\ \text { DI2 } & 56.1191 \\ \text { DI3 } & 119.008 \\ \text { DI4 } & -120.7283 \\ \text { DI5 } & 53.296 \mathrm{~F} \\ \text { DI6 } & 194.3855 \\ \text { DI7 } & -20.0389 \\ \text { DI8 } & 120.5773 \\ \text { DI9 } & 120.8974 \\ \text { DI10 } & -118.9618 \\ & \end{array}$

$M P 2$
$\mathrm{P}$

H 1 PH1

C 1 PH2 $2 \mathrm{HPH}$

C 1 PC 2 CPH 3 DI 1

H 4 CH1 1 HCP 2 DI2

$\mathrm{H} 4 \mathrm{CH} 21 \mathrm{HCP} 25 \mathrm{DI} 3$

$\mathrm{H} 4 \mathrm{CH} 31 \mathrm{HCP} 35$ DI4

$\mathrm{X} 3 \mathrm{CH} 41 \mathrm{HCP} 42$ DI5

H 3 CH5 1 HCP5 8 DI6

C 3 CH6 1 HCP6 8 DI7
H 10 CH7 3 HCC1 8 DI8

H 10 CH8 3 HCC2 11 DI9

H 10 CH9 3 HCC3 11 DI10

$\begin{array}{lc}\text { PH1 } & 1.465 \\ \text { PH2 } & 1.7496 \\ \text { PC } & 1.8794 \\ \text { CH1 } & 1.0959 \\ \text { CH2 } & 1.0911 \\ \text { CH3 } & 1.0925 \\ \text { CH4 } & 1.0968 \\ \text { CH5 } & 1.0882 \\ \text { CH6 } & 1.5114 \\ \text { CH7 } & 1.0965 \\ \text { CH8 } & 1.1116 \\ \text { CH9 } & 1.0952 \\ \text { HPH } & 106.6258 \\ \text { CPH } & 90.4821 \\ \text { HCP } & 109.6697 \\ \text { HCP2 } & 109.0471 \\ \text { HCP3 } & 112.2774 \\ \text { HCP4 } & 110.3484 \\ \text { HCP5 } & 113.1531 \\ \text { HCP6 } & 120.3808 \\ \text { HCC1 } & 111.3385 \\ \text { HCC2 } & 114.0734 \\ \text { HCC3 } & 112.492 \\ \text { DI1 } & 105.5798 \\ \text { DI2 } & 56.6759 \\ \text { DI3 } & 119.2813 \\ \text { DI4 } & -120.4695 \\ \text { DI5 } & 53.296 \\ \text { DI6 } & 196.4097 \\ \text { DI7 } & -21.2089 \\ \text { DI8 } & 116.8944 \\ \text { DI9 } & 120.9985 \\ \text { DI10 } & -118.9513\end{array}$

$\mathrm{C}_{2} \mathrm{H}_{5} \mathrm{PHCH}_{3}{ }^{-}$

B3LYP

$\mathrm{C}$

$\mathrm{C} 1 \mathrm{CC}$

C 2 CC $11 \mathrm{CCC}$

H 1 CH1 2 HCC1 3 DI 1

$\mathrm{H} 1 \mathrm{CH} 22 \mathrm{HCC} 24 \mathrm{DI} 2$

$\mathrm{H} 2 \mathrm{CH} 31 \mathrm{HCC} 34$ DI 3

H 3 CH4 2 HCC4 6 DI4

P 3 CH5 2 HCC5 7 DI5

C 8 PC 3 PCC 2 DI6

H 8 PH 3 HCP 9 DI7

H 9 CH6 8 HCP2 10 DI8

H 9 CH7 8 HCP3 11 DI9

H 9 CH8 8 HCP4 11 DI10

$\begin{array}{ll}\mathrm{CC} & 1.3813 \\ \mathrm{CC} 1 & 1.4155 \\ \mathrm{CH} 1 & 1.0895 \\ \mathrm{CH} 2 & 1.087 \\ \mathrm{CH} 3 & 1.0967 \\ \mathrm{CH} 4 & 1.0936 \\ \mathrm{CH} 5 & 1.7672 \\ \mathrm{PC} & 1.9037 \\ \mathrm{PH} & 1.4496 \\ \mathrm{CH} 6 & 1.0975 \\ \mathrm{CH} 7 & 1.1013 \\ \mathrm{CH} 8 & 1.0961 \\ \mathrm{CCC} & 130.2577 \\ \mathrm{HCC} 1 & 121.889 \\ \mathrm{HCC} 2 & 121.035 \\ \mathrm{HCC} 3 & 115.2668 \\ \mathrm{HCC} 4 & 118.117 \\ \mathrm{HCC} 5 & 121.6427 \\ \text { PCC } & 106.7204 \\ \mathrm{HCP} & 104.1765 \\ \mathrm{HCP} 2 & 112.1596 \\ \mathrm{HCP} 3 & 109.859\end{array}$

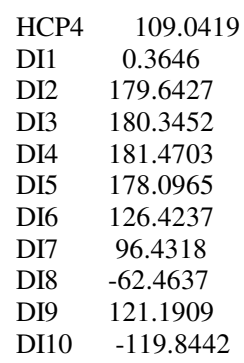

$M P 2$

$\mathrm{C}$

$\mathrm{C} 1 \mathrm{CC}$

$\mathrm{C} 2 \mathrm{CC} 11 \mathrm{CCC}$

H 1 CH1 2 HCC1 3 DI

$\mathrm{H} 1 \mathrm{CH} 22 \mathrm{HCC} 24$ DI2

$\mathrm{H} 2 \mathrm{CH} 31 \mathrm{HCC} 34$ DI3

H 3 CH4 2 HCC4 6 DI4

P 3 CH5 2 HCC5 7 DI5

C 8 PC 3 PCC 2 DI6

$\mathrm{H} 8 \mathrm{PH} 3 \mathrm{HCP} 9 \mathrm{DI} 7$

H 9 CH6 8 HCP2 10 DI8

$\mathrm{H} 9 \mathrm{CH} 78$ HCP3 11 DI9

H 9 CH8 8 HCP4 11 DI10

$\begin{array}{lc}\text { CC } & 1.3805 \\ \text { CC1 } & 1.4164 \\ \text { CH1 } & 1.0851 \\ \text { CH2 } & 1.0828 \\ \text { CH3 } & 1.0933 \\ \text { CH4 } & 1.0902 \\ \text { CH5 } & 1.7592 \\ \text { PC } & 1.8786 \\ \text { PH } & 1.4334 \\ \text { CH6 } & 1.0924 \\ \text { CH7 } & 1.0962 \\ \text { CH8 } & 1.0909 \\ \text { CCC } & 129.9061 \\ \text { HCC1 } & 121.7513 \\ \text { HCC2 } & 120.8639 \\ \text { HCC3 } & 115.4658 \\ \text { HCC4 } & 118.221 \\ \text { HCC5 } & 120.8065 \\ \text { PCC } & 105.4151 \\ \text { HCP } & 105.1822 \\ \text { HCP2 } & 111.9282 \\ \text { HCP3 } & 110.3445 \\ \text { HCP4 } & 108.848 \\ \text { DI1 } & -0.1333 \\ \text { DI2 } & 178.9084 \\ \text { DI3 } & 180.5044 \\ \text { DI } & 183.6327 \\ \text { DI5 } & 172.1273 \\ \text { DI6 } & 131.6475 \\ \text { DI7 } & 96.8672 \\ \text { DI8 } & -61.7486 \\ \text { DI9 } & 121.2313 \\ \text { DI10 } & -119.6437 \\ & \end{array}$

$\mathrm{CH}_{3} \mathrm{P}\left(\mathrm{CH}_{3}\right)_{2}$

B3LYP
P
C 1 PH1
C 1 PH2 2 HPH
C 1 PC 2 CPH 3 DI 1
H 4 CH1 1 HCP 2 DI2
H 4 CH2 1 HCP2 5 DI3
H 4 CH3 1 HCP3 5 DI4
H 3 CH4 1 HCP4 2 DI5
H 3 CH5 1 HCP5 8 DI6
H 3 CH6 1 HCP6 8 DI7
H 2 CH7 1 HCP7 3 DI8
H 2 CH8 1 HCP8 11 DI9
H 2 CH9 1 HCP9 11 DI10




$\begin{array}{lc}\text { PH1 } & 1.8664 \\ \text { PH2 } & 1.8664 \\ \text { PC } & 1.8665 \\ \text { CH1 } & 1.0948 \\ \text { CH2 } & 1.0948 \\ \text { CH3 } & 1.0971 \\ \text { CH4 } & 1.0948 \\ \text { CH5 } & 1.097 \\ \text { CH6 } & 1.0947 \\ \text { CH7 } & 1.0948 \\ \text { CH8 } & 1.0947 \\ \text { CH9 } & 1.097 \\ \text { HPH } & 99.4106 \\ \text { CPH } & 99.4291 \\ \text { HCP } & 109.5291 \\ \text { HCP2 } & 109.5324 \\ \text { HCP3 } & 112.6053 \\ \text { HCP4 } & 109.5465 \\ \text { HCP5 } & 112.5465 \\ \text { HCP6 } & 109.5547 \\ \text { HCP7 } & 109.5375 \\ \text { HCP8 } & 109.5312 \\ \text { HCP9 } & 112.5738 \\ \text { DI1 } & 101.3148 \\ \text { DI2 } & 70.5023 \\ \text { DI3 } & 117.746 \\ \text { DI4 } & -121.1179 \\ \text { DI5 } & -70.3579 \\ \text { DI6 } & 121.0767 \\ \text { DI7 } & -117.7954 \\ \text { DI8 } & 70.6878 \\ \text { DI9 } & 117.7485 \\ \text { DI10 } & -121.1232 \\ & \end{array}$

$M P 2$

$\mathrm{P}$

C 1 PH1

C 1 PH2 $2 \mathrm{HPH}$

C 1 PC 2 CPH 3 DI1

H 4 CH1 1 HCP 2 DI2

$\mathrm{H} 4 \mathrm{CH} 21 \mathrm{HCP} 25 \mathrm{DI} 3$

$\mathrm{H} 4 \mathrm{CH} 31$ HCP3 5 DI4

H 3 CH4 1 HCP4 2 DI5

H 3 CH5 1 HCP5 8 DI6

H 3 CH6 1 HCP6 8 DI7

$\mathrm{H} 2 \mathrm{CH} 71$ HCP7 3 DI8

H 2 CH8 1 HCP8 11 DI9

H 2 CH9 1 HCP9 11 DI10

$\begin{array}{lc}\text { PH1 } & 1.8494 \\ \text { PH2 } & 1.8494 \\ \text { PC } & 1.8494 \\ \text { CH1 } & 1.0902 \\ \text { CH2 } & 1.0902 \\ \text { CH3 } & 1.0926 \\ \text { CH4 } & 1.0902 \\ \text { CH5 } & 1.0926 \\ \text { CH6 } & 1.0902 \\ \text { CH7 } & 1.0902 \\ \text { CH8 } & 1.0902 \\ \text { CH9 } & 1.0926 \\ \text { HPH } & 99.0775 \\ \text { CPH } & 99.0765 \\ \text { HCP } & 109.8085 \\ \text { HCP2 } & 109.8054 \\ \text { HCP3 } & 111.8903 \\ \text { HCP4 } & 109.805 \\ \text { HCP5 } & 111.8893 \\ \text { HCP6 } & 109.8092 \\ \text { HCP7 } & 109.8098 \\ \text { HCP8 } & 109.8065 \\ \text { HCP9 } & 111.8873 \\ \text { DI1 } & 100.7953 \\ \text { DI2 } & 70.5224 \\ \text { DI3 } & 118.1119 \\ \text { DI4 } & -120.9436\end{array}$

$\begin{array}{lc}\text { DI5 } & -70.5029 \\ \text { DI6 } & 120.9407 \\ \text { DI7 } & -118.1111 \\ \text { DI8 } & 70.5054 \\ \text { DI9 } & 118.1146 \\ \text { DI10 } & -120.9414\end{array}$

$\mathrm{CH}_{2} \mathrm{P}\left(\mathrm{CH}_{3}\right)_{2}^{-}$

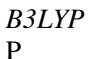

C 1 PC1

C 1 PC2 2 CPC 1

H 2 CH1 1 HCP1 3 DI

$\mathrm{H} 2 \mathrm{CH} 21 \mathrm{HCP} 24$ DI2

$\mathrm{H} 2 \mathrm{CH} 31 \mathrm{HCP} 34 \mathrm{DI} 3$

H 3 CH4 1 HCP4 2 DI 4

H 3 CH5 1 HCP5 7 DI5

H 3 CH6 1 HCP6 7 DI6

C 1 PC3 2 CPC2 3 DI7

$\mathrm{H} 10 \mathrm{CH} 71 \mathrm{HCP} 72 \mathrm{DI} 8$

H 10 CH8 1 HCP8 11 DI9

$\begin{array}{lc}\text { PC1 } & 1.9201 \\ \text { PC2 } & 1.903 \\ \text { CH1 } & 1.102 \\ \text { CH2 } & 1.1014 \\ \text { CH3 } & 1.0956 \\ \text { CH4 } & 1.1005 \\ \text { CH5 } & 1.0949 \\ \text { CH6 } & 1.1011 \\ \text { PC3 } & 1.7421 \\ \text { CH7 } & 1.0872 \\ \text { CH8 } & 1.0943 \\ \text { CPC1 } & 93.4002 \\ \text { HCP1 } & 111.9895 \\ \text { HCP2 } & 111.1558 \\ \text { HCP3 } & 108.0444 \\ \text { HCP4 } & 111.6362 \\ \text { HCP5 } & 108.341 \\ \text { HCP6 } & 111.1138 \\ \text { CPC2 } & 108.2796 \\ \text { HCP7 } & 118.0968 \\ \text { HCP8 } & 123.2154 \\ \text { DI1 } & -61.977 \\ \text { DI2 } & 121.9277 \\ \text { DI3 } & -119.6206 \\ \text { DI4 } & 59.4367 \\ \text { DI5 } & 119.9109 \\ \text { DI6 } & -121.5206 \\ \text { DI7 } & -107.0364 \\ \text { DI8 } & -117.8352 \\ \text { DI9 } & 163.1487 \\ & \\ & \end{array}$

$M P 2$

$\mathrm{P}$

C 1 PC1

C 1 PC2 2 CPC1

H 2 CH1 1 HCP1 3 DI 1

$\mathrm{H} 2 \mathrm{CH} 21 \mathrm{HCP} 24$ DI2

$\mathrm{H} 2 \mathrm{CH} 31 \mathrm{HCP} 34$ DI 3

$\mathrm{H} 3 \mathrm{CH} 41 \mathrm{HCP} 42$ DI4

H 3 CH5 1 HCP5 7 DI5

H 3 CH6 1 HCP6 7 DI6

C 1 PC3 2 CPC2 3 DI7

H 10 CH7 1 HCP7 2 DI8

H 10 CH8 1 HCP8 11 DI9

$\begin{array}{ll}\text { PC1 } & 1.8935 \\ \text { PC2 } & 1.8814 \\ \text { CH1 } & 1.0967 \\ \text { CH2 } & 1.0966 \\ \text { CH3 } & 1.0907 \\ \text { CH4 } & 1.0959 \\ \text { CH5 } & 1.0901 \\ \text { CH6 } & 1.0963 \\ \text { PC3 } & 1.7377\end{array}$

$\begin{array}{lc}\text { CH7 } & 1.083 \\ \text { CH8 } & 1.0904 \\ \text { CPC1 } & 93.1507 \\ \text { HCP1 } & 111.8178 \\ \text { HCP2 } & 110.4473 \\ \text { HCP3 } & 108.6195 \\ \text { HCP4 } & 111.6894 \\ \text { HCP5 } & 108.7828 \\ \text { HCP6 } & 110.2731 \\ \text { CPC2 } & 107.6644 \\ \text { HCP7 } & 118.3202 \\ \text { HCP8 } & 122.4874 \\ \text { DI1 } & -61.1976 \\ \text { DI2 } & 121.5359 \\ \text { DI3 } & -119.944 \\ \text { DI4 } & 58.6915 \\ \text { DI5 } & 120.1633 \\ \text { DI6 } & -121.3747 \\ \text { DI7 } & -106.4349 \\ \text { DI8 } & -119.7728 \\ \text { DI9 } & 162.938\end{array}$

$B 3 L Y P /(D E L)$

$\mathrm{P}$

C 1 PH 1

C 1 PH2 $2 \mathrm{HPH}$

C 1 PC 2 CPH 3 DI1

$\mathrm{H} 4 \mathrm{CH} 11$ HCP 2 DI2

$\mathrm{H} 4 \mathrm{CH} 21 \mathrm{HCP} 25 \mathrm{DI} 3$

$\mathrm{H} 4 \mathrm{CH} 31 \mathrm{HCP} 35$ DI4

$\mathrm{H} 3 \mathrm{CH} 41 \mathrm{HCP} 42$ DI5

H 3 CH5 1 HCP5 8 DI6

H 3 CH6 1 HCP6 8 DI7

$\mathrm{H} 2 \mathrm{CH} 71 \mathrm{HCP} 73$ DI8

H 2 CH9 1 HCP9 11 DI10

PH1 1.870692

PH2 1.866985

PC 1.866849

CH1 1.097252

CH2 1.095978

CH3 1.102851

CH4 1.097285

CH5 1.102847

CH6 1.096125

CH7 1.110935

CH9 1.110964

HPH 99.602

$\begin{array}{ll}\mathrm{CPH} & 99.539\end{array}$

HCP 109.176

HCP2 111.305

HCP3 109.556

HCP4 109.220

HCP5 109.700

HCP6 111.180

HCP7 105.870

HCP9 105.925

DI1 100.002

DI2 78.395

DI3 119.344

DI4 -118.354

DI5 -78.534

DI6 118.567

DI7 -119.289

DI8 185.128

DI10 -110.111

$\mathrm{C}_{2} \mathrm{H}_{4} \mathrm{P}\left(\mathrm{CH}_{3}\right)_{2}{ }^{-}$

B3LYP

$\mathrm{P}$

C 1 PH1

C 1 PH2 $2 \mathrm{HPH}$

C 1 PC 2 CPH 3 DI 1

H 4 CH1 1 HCP 2 DI2

$\mathrm{H} 4 \mathrm{CH} 21 \mathrm{HCP} 25 \mathrm{DI} 3$ 


\begin{tabular}{|c|c|c|c|c|c|}
\hline \multicolumn{2}{|c|}{ H 4 CH3 1 HCP3 5 DI4 } & $\mathrm{CH} 3$ & 1.0961 & $\mathrm{HCC} 4$ & 118.2845 \\
\hline \multicolumn{2}{|c|}{ H 3 CH4 1 HCP4 2 DI5 } & $\mathrm{CH} 4$ & 1.0918 & HCC4 & 118.2845 \\
\hline \multicolumn{2}{|c|}{ H 3 CH5 1 HCP5 8 DI6 } & $\mathrm{CH} 5$ & 1.0973 & HCC5 & 122.5693 \\
\hline \multicolumn{2}{|c|}{ H 3 CH6 1 HCP6 8 DI7 } & $\mathrm{CH} 6$ & 1.0978 & PCC & 104.8324 \\
\hline \multicolumn{2}{|c|}{ X 2 CH7 1 HCP7 3 DI8 } & $\mathrm{CH} 7$ & 1.0975 & HCP & 104.814 \\
\hline \multicolumn{2}{|c|}{ H 2 CH8 1 HCP8 11 DI9 } & $\mathrm{CH} 8$ & 1.0957 & HCP2 & 111.5114 \\
\hline \multicolumn{2}{|c|}{ C 2 CH9 1 HCP9 11 DI10 } & $\mathrm{CH} 9$ & 1.5128 & HCP3 & 111.2369 \\
\hline \multicolumn{2}{|c|}{ H 13 CH10 2 HCC1 11 DI1 1} & CH10 & 1.099 & HCP4 & 108.5761 \\
\hline H $13 \mathrm{C}$ & H11 2 HCC2 14 DI12 & CH11 & 1.1065 & HCP5 & 111.5142 \\
\hline H $13 \mathrm{C}$ & H12 2 HCC3 14 DI13 & CH12 & 1.0957 & HCP6 & 111.2491 \\
\hline & & $\mathrm{HPH}$ & 110.9901 & HCP7 & 108.5734 \\
\hline PH1 & 1.7419 & $\mathrm{CPH}$ & 102.5902 & DI1 & -0.0057 \\
\hline PH2 & 1.9373 & $\mathrm{HCP}$ & 109.4076 & DI2 & 180.0222 \\
\hline PC & 1.8949 & $\mathrm{HCP} 2$ & 111.3461 & DI3 & 179.994 \\
\hline $\mathrm{CH} 1$ & 1.0946 & $\mathrm{HCP} 3$ & 110.0109 & DI4 & 179.9744 \\
\hline $\mathrm{CH} 2$ & 1.0997 & HCP4 & 108.385 & DI5 & 180.0691 \\
\hline $\mathrm{CH} 3$ & 1.1008 & HCP5 & 110.6734 & DI6 & 130.3663 \\
\hline $\mathrm{CH} 4$ & 1.0962 & HCP6 & 111.9039 & DI7 & 99.1072 \\
\hline CH5 & 1.1018 & HCP7 & 106.3457 & DI8 & -58.5758 \\
\hline CH6 & 1.1028 & HCP8 & 117.8258 & DI9 & 121.5236 \\
\hline $\mathrm{CH} 7$ & $1.0975 \mathrm{~F}$ & НCP9 & 120.0026 & DI10 & -119.0909 \\
\hline $\mathrm{CH} 8$ & 1.0983 & $\mathrm{HCC} 1$ & 112.1422 & DI11 & 58.546 \\
\hline $\mathrm{CH} 9$ & 1.5131 & $\mathrm{HCC} 2$ & 116.227 & DI12 & -121.5271 \\
\hline $\mathrm{CH} 10$ & 1.1071 & HCC3 & 109.6989 & DI13 & 119.0822 \\
\hline CH11 & 1.1149 & DI1 & 98.3085 & & \\
\hline $\mathrm{CH} 12$ & 1.101 & DI2 & 73.8959 & $M P 2$ & \\
\hline $\mathrm{HPH}$ & 110.4328 & DI3 & 120.5586 & $\mathrm{C}$ & \\
\hline $\mathrm{CPH}$ & 103.6219 & DI4 & -118.7343 & $\mathrm{C} 1 \mathrm{CC}$ & \\
\hline $\mathrm{HCP}$ & 108.7928 & DI5 & -69.2362 & $\mathrm{C} 2 \mathrm{CC}$ & $11 \mathrm{CCC}$ \\
\hline HCP2 & 111.4221 & DI6 & 118.762 & $\mathrm{H} 1 \mathrm{CH}$ & 112 HCC1 3 DI1 \\
\hline HCP3 & 110.8385 & DI7 & -119.3343 & $\mathrm{H} 1 \mathrm{CH}$ & 222 HCC2 4 DI2 \\
\hline $\mathrm{HCP} 4$ & 108.0228 & DI8 & 196.4162 & $\mathrm{H} 2 \mathrm{CH}$ & $3131 \mathrm{HCC} 34$ DI3 \\
\hline HCP5 & 111.2816 & DI9 & 115.633 & $\mathrm{H} 3 \mathrm{CH}$ & 42 HCC4 6 DI4 \\
\hline HCP6 & 112.0544 & DI10 & -93.8439 & P $3 \mathrm{CH}$ & 52 HCC5 7 DI5 \\
\hline $\mathrm{HCP} 7$ & $106.3457 \mathrm{~F}$ & DI11 & 41.7488 & C 8 PC & 3 PCC 2 DI6 \\
\hline HCP8 & 118.9881 & DI12 & 121.6791 & C 8 PC & 23 HCP 9 DI7 \\
\hline $\mathrm{HCP} 9$ & 121.4973 & DI13 & -118.1583 & $\mathrm{H} 9 \mathrm{CH}$ & 168 HCP2 10 DI8 \\
\hline $\mathrm{HCC} 1$ & 112.7076 & & & $\mathrm{H} 9 \mathrm{CH}$ & 178 HCP3 11 DI9 \\
\hline $\mathrm{HCC} 2$ & 116.1162 & $\mathrm{C}_{3} \mathrm{H}_{4} \mathrm{P}$ & $\left(\mathrm{CH}_{3}\right)_{2}^{-}$ & $\mathrm{H} 9 \mathrm{CH}$ & 88 HCP4 11 DI10 \\
\hline $\mathrm{HCC} 3$ & 110.3648 & & & $\mathrm{H} 10 \mathrm{C}$ & H9 8 HCP5 9 DI11 \\
\hline & 98.6802 & $B 3 L Y P$ & & H $10 \mathrm{C}$ & H10 8 HCP6 14 DI12 \\
\hline & 71.847 & $\mathrm{C}$ & & H $10 \mathrm{C}$ & H11 8 HCP7 14 DI13 \\
\hline DI3 & 120.2516 & $\mathrm{C} 1 \mathrm{CO}$ & & & \\
\hline & -118.7495 & $\mathrm{C} 2 \mathrm{CC}$ & $11 \mathrm{CCC}$ & $\mathrm{CC}$ & 1.3806 \\
\hline & -69.5593 & $\mathrm{H} 1 \mathrm{CH}$ & I1 2 HCC1 3 DI1 & $\mathrm{CC} 1$ & 1.4161 \\
\hline DI6 & 118.6348 & $\mathrm{H} 1 \mathrm{CH}$ & I2 2 HCC2 4 DI2 & $\mathrm{CH} 1$ & 1.0852 \\
\hline DI7 & -119.2267 & $\mathrm{H} 2 \mathrm{CH}$ & I3 1 HCC 34 DI3 & $\mathrm{CH} 2$ & 1.0829 \\
\hline & $196.4162 \mathrm{~F}$ & $\mathrm{H} 3 \mathrm{CH}$ & I4 2 HCC4 6 DI4 & $\mathrm{CH} 3$ & 1.0932 \\
\hline DI9 & 113.4463 & P $3 \mathrm{CH}$ & 52 HCC5 7 DI5 & $\mathrm{CH} 4$ & 1.093 \\
\hline DI10 & -88.6908 & C 8 PC & 3 PCC 2 DI6 & CH5 & 1.756 \\
\hline DI11 & 38.8323 & C 8 PC & 23 HCP 9 DI7 & $\mathrm{PC}$ & 1.8766 \\
\hline DI12 & 121.3274 & $\mathrm{H} 9 \mathrm{CH}$ & 168 HCP2 10 DI8 & $\mathrm{PC} 2$ & 1.8766 \\
\hline DI13 & -118.5005 & $\mathrm{H} 9 \mathrm{CH}$ & 178 HCP3 11 DI9 & $\mathrm{CH} 6$ & 1.0953 \\
\hline & & $\mathrm{H} 9 \mathrm{CH}$ & 188 HCP4 11 DI10 & $\mathrm{CH} 7$ & 1.0952 \\
\hline MP2 & & H $10 \mathrm{C}$ & H9 8 HCP5 9 DI11 & $\mathrm{CH} 8$ & 1.0904 \\
\hline $\mathrm{P}$ & & H $10 \mathrm{C}$ & H10 8 HCP6 14 DI12 & $\mathrm{CH} 9$ & 1.0953 \\
\hline C $1 \mathrm{PH}$ & & $\mathrm{H} 10 \mathrm{C}$ & H11 8 HCP7 14 DI13 & $\mathrm{CH} 10$ & 1.0952 \\
\hline C $1 \mathrm{PH}$ & $22 \mathrm{HPH}$ & & & CH11 & 1.0904 \\
\hline C 1 PC & 2 CPH 3 DI1 & $\mathrm{CC}$ & 1.3815 & $\mathrm{CCC}$ & 130.0141 \\
\hline $\mathrm{H} 4 \mathrm{Cl}$ & $11 \mathrm{HCP} 2 \mathrm{DI} 2$ & $\mathrm{CC} 1$ & 1.4154 & $\mathrm{HCC} 1$ & 121.7806 \\
\hline $\mathrm{H} 4 \mathrm{Cl}$ & 21 HCP2 5 DI3 & $\mathrm{CH} 1$ & 1.0895 & $\mathrm{HCC} 2$ & 120.8463 \\
\hline $\mathrm{H} 4 \mathrm{Cl}$ & 31 HCP3 5 DI4 & $\mathrm{CH} 2$ & 1.087 & HCC 3 & 115.4432 \\
\hline $\mathrm{H} 3 \mathrm{Cl}$ & $41 \mathrm{HCP} 42$ DI5 & $\mathrm{CH} 3$ & 1.0966 & $\mathrm{HCC} 4$ & 118.6229 \\
\hline $\mathrm{H} 3 \mathrm{Cl}$ & 51 HCP5 8 DI6 & $\mathrm{CH} 4$ & 1.0962 & HCC5 & 121.9469 \\
\hline $\mathrm{H} 3 \mathrm{Cl}$ & 61 HCP6 8 DI7 & CH5 & 1.7652 & PCC & 104.4617 \\
\hline $\mathrm{X} 2 \mathrm{Cl}$ & 7 1 HCP7 3 DI8 & $\mathrm{PC}$ & 1.8976 & $\mathrm{HCP}$ & 104.457 \\
\hline $\mathrm{H} 2 \mathrm{Cl}$ & 81 HCP8 11 DI9 & $\mathrm{PC} 2$ & 1.8973 & HCP2 & 110.881 \\
\hline $\mathrm{C} 2 \mathrm{Cl}$ & 91 HCP9 11 DI10 & $\mathrm{CH} 6$ & 1.1 & HCP3 & 111.2883 \\
\hline H $13 \mathrm{C}$ & H10 2 HCC1 11 DI11 & $\mathrm{CH} 7$ & 1.0998 & $\mathrm{HCP} 4$ & 108.8626 \\
\hline H $13 \mathrm{C}$ & H11 2 HCC2 14 DI12 & $\mathrm{CH} 8$ & 1.0949 & HCP5 & 110.8789 \\
\hline $\mathrm{H} 13 \mathrm{C}$ & H12 2 HCC3 14 DI13 & $\mathrm{CH} 9$ & 1.1001 & HCP6 & 111.2927 \\
\hline & & $\mathrm{CH} 10$ & 1.0998 & HCP7 & 108.8631 \\
\hline PH1 & 1.74 & CH11 & 1.0949 & DI1 & -0.0003 \\
\hline PH2 & 1.9138 & $\mathrm{CCC}$ & 130.3733 & DI2 & 180.0173 \\
\hline $\mathrm{PC}$ & 1.8708 & $\mathrm{HCC} 1$ & 121.9157 & DI3 & 179.9946 \\
\hline $\mathrm{CH} 1$ & 1.0897 & $\mathrm{HCC} 2$ & 120.999 & DI4 & 179.9893 \\
\hline $\mathrm{CH} 2$ & 1.095 & $\mathrm{HCC} 3$ & 115.2251 & DI5 & 180.0409 \\
\hline
\end{tabular}




$\begin{array}{lc}\text { DI6 } & 130.6325 \\ \text { DI7 } & 98.6582 \\ \text { DI8 } & -58.7015 \\ \text { DI9 } & 121.3063 \\ \text { DI10 } & -119.0736 \\ \text { DI11 } & 58.7073 \\ \text { DI12 } & -121.3073 \\ \text { DI13 } & 119.0705\end{array}$

$\mathrm{CH}_{3} \mathrm{PO}\left(\mathrm{CH}_{3}\right)_{2}$

B3LYP

$\mathrm{P}$

C 1 PH1

C 1 PH2 $2 \mathrm{HPH}$

C 1 PC 2 CPH 3 DI1

H 4 CH1 1 HCP 2 DI2

$\mathrm{H} 4 \mathrm{CH} 21 \mathrm{HCP} 25 \mathrm{DI} 3$

$\mathrm{H} 4 \mathrm{CH} 31 \mathrm{HCP} 35 \mathrm{DI} 4$

H 3 CH4 1 HCP4 2 DI5

H 3 CH5 1 HCP5 8 DI6

H 3 CH6 1 HCP6 8 DI7

O 1 PO 2 OPH 3 DI8

H 2 CH7 1 HCP7 3 DI9

H 2 CH8 1 HCP8 12 DI10

H 2 CH9 1 HCP9 12 DI1 1

$\begin{array}{lc}\text { PH1 } & 1.834 \\ \text { PH2 } & 1.8336 \\ \text { PC } & 1.8336 \\ \text { CH1 } & 1.0937 \\ \text { CH2 } & 1.0937 \\ \text { CH3 } & 1.0947 \\ \text { CH4 } & 1.0938 \\ \text { CH5 } & 1.0946 \\ \text { CH6 } & 1.0938 \\ \text { CH7 } & 1.0938 \\ \text { CH8 } & 1.0939 \\ \text { CH9 } & 1.0946 \\ \text { PO } & 1.5058 \\ \text { HPH } & 104.8521 \\ \text { CPH } & 104.8587 \\ \text { HCP } & 108.9003 \\ \text { HCP2 } & 109.0184 \\ \text { HCP3 } & 112.0178 \\ \text { HCP4 } & 108.9324 \\ \text { HCP5 } & 112.0015 \\ \text { HCP6 } & 108.9858 \\ \text { OPH } & 113.7708 \\ \text { HCP7 } & 108.852 \\ \text { HCP8 } & 109.0477 \\ \text { HCP9 } & 112.0701 \\ \text { DI1 } & 110.1677 \\ \text { DI2 } & 66.6278 \\ \text { DI3 } & 117.9036 \\ \text { DI4 } & -121.0181 \\ \text { DI5 } & -65.9976 \\ \text { DI6 } & 121.0245 \\ \text { DI7 } & -117.8924 \\ \text { DI8 } & -124.911 \\ \text { DI9 } & 67.8386 \\ \text { DI10 } & 117.8357 \\ \text { DI11 } & -121.0429 \\ & \end{array}$

$M P 2$

C 1 PH1

C 1 PH2 $2 \mathrm{HPH}$

C 1 PC 2 CPH 3 DI 1

$\mathrm{H} 4 \mathrm{CH} 11 \mathrm{HCP} 2 \mathrm{DI} 2$

$\mathrm{H} 4 \mathrm{CH} 21 \mathrm{HCP} 25 \mathrm{DI} 3$

$\mathrm{H} 4 \mathrm{CH} 31 \mathrm{HCP} 35$ DI4

H 3 CH4 1 HCP4 2 DI5

H 3 CH5 1 HCP5 8 DI6

H 3 CH6 1 HCP6 8 DI7

O 1 PO 2 OPH 3 DI8
H 2 CH7 1 HCP7 3 DI9

H 2 CH8 1 HCP8 12 DI10

H 2 CH9 1 HCP9 12 DI1 1

PH1 1.8187

PH2 1.8187

PC $\quad 1.8187$

CH1 1.0894

$\mathrm{CH} 21.0894$

CH3 1.0903

CH4 1.0894

CH5 1.0903

CH6 1.0894

CH7 1.0894

CH8 1.0894

CH9 1.0903

PO $\quad 1.5117$

HPH 104.4665

CPH 104.4694

HCP 109.1091

HCP2 109.1346

HCP3 111.5921

HCP4 109.1316

HCP5 111.5961

HCP6 109.1077

OPH 114.1024

HCP7 109.11

HCP8 109.1357

HCP9 111.5899

DI1 109.4594

DI2 66.3635

DI3 118.1496

DI4 -120.9164

DI5 -65.9983

DI6 120.9324

DI7 -118.1461

DI8 -125.2663

DI9 66.3084

DI10 118.1562

DI1 -120.9104

$\mathrm{CH}_{2} \mathrm{PO}\left(\mathrm{CH}_{3}\right)_{2}^{-}$

B3LYP

$\mathrm{P}$

C 1 PH1

C 1 PH2 $2 \mathrm{HPH}$

C 1 PC 2 CPH 3 DI1

$\mathrm{H} 4 \mathrm{CH} 11$ HCP 2 DI2

$\mathrm{H} 4 \mathrm{CH} 21 \mathrm{HCP} 25$ DI3

$\mathrm{H} 4 \mathrm{CH} 31 \mathrm{HCP} 35$ DI4

H 3 CH4 1 HCP4 2 DI5

H 3 CH5 1 HCP5 8 DI6

H 3 CH6 1 HCP6 8 DI7

O 1 PO 2 OPH 3 DI8

$\mathrm{H} 2 \mathrm{CH} 71 \mathrm{HCP} 73 \mathrm{DI} 9$

H 2 CH8 1 HCP8 12 DI10

$\mathrm{X} 2 \mathrm{CH} 91$ HCP9 12 DI11

$\begin{array}{ll}\text { PH1 } & 1.7357 \\ \text { PH2 } & 1.8622 \\ \text { PC } & 1.8622 \\ \text { CH1 } & 1.0961 \\ \text { CH2 } & 1.0959 \\ \text { CH3 } & 1.0941 \\ \text { CH4 } & 1.0961 \\ \text { CH5 } & 1.0941 \\ \text { CH6 } & 1.0958 \\ \text { CH7 } & 1.0958 \\ \text { CH8 } & 1.0958 \\ \text { CH9 } & 1.0946 \\ \text { PO } & 1.5406 \\ \text { HPH } & 105.3515 \\ \text { CPH } & 105.3867 \\ \text { HCP } & 108.0891 \\ \text { HCP2 } & 109.5498 \\ \text { HCP3 } & 111.812\end{array}$

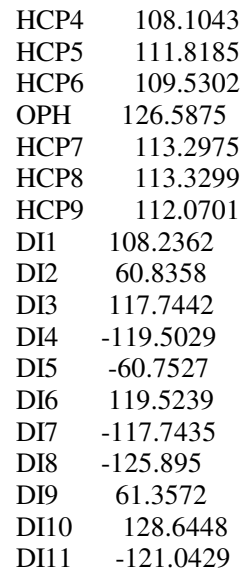

MP2

$\mathrm{P}$

C 1 PH1

C 1 PH2 $2 \mathrm{HPH}$

C 1 PC 2 CPH 3 DI 1

$\mathrm{H} 4 \mathrm{CH} 11$ HCP 2 DI2

$\mathrm{H} 4 \mathrm{CH} 21 \mathrm{HCP} 25 \mathrm{DI} 3$

$\mathrm{H} 4 \mathrm{CH} 31 \mathrm{HCP} 35 \mathrm{DI} 4$

H 3 CH4 1 HCP4 2 DI5

H 3 CH5 1 HCP5 8 DI6

H 3 CH6 1 HCP6 8 DI7

O 1 PO 2 OPH 3 DI8

H 2 CH7 1 HCP7 3 DI9

H 2 CH8 1 HCP8 12 DI10

X 2 CH9 1 HCP9 12 DI11

\begin{tabular}{|c|c|}
\hline PH1 & 1.7263 \\
\hline PH2 & 1.8444 \\
\hline $\mathrm{PC}$ & 1.8444 \\
\hline $\mathrm{CH} 1$ & 1.0915 \\
\hline $\mathrm{CH} 2$ & 1.0913 \\
\hline $\mathrm{CH} 3$ & 1.0895 \\
\hline $\mathrm{CH} 4$ & 1.0915 \\
\hline CH5 & 1.0895 \\
\hline CH6 & 1.0913 \\
\hline $\mathrm{CH} 7$ & 1.091 \\
\hline $\mathrm{CH} 8$ & 1.091 \\
\hline CH9 & 1.0946 \\
\hline $\mathrm{PO}$ & 1.5467 \\
\hline $\mathrm{HPH}$ & 105.2774 \\
\hline $\mathrm{CPH}$ & 105.3011 \\
\hline $\mathrm{HCP}$ & 108.2948 \\
\hline $\mathrm{HCP} 2$ & 109.3768 \\
\hline HCP3 & 111.5498 \\
\hline HCP4 & 108.2975 \\
\hline HCP5 & 111.5558 \\
\hline HCP6 & 109.3681 \\
\hline $\mathrm{OPH}$ & 126.6012 \\
\hline $\mathrm{HCP} 7$ & 113.3983 \\
\hline HCP8 & 113.4146 \\
\hline HCP9 & 112.0701 \\
\hline DI1 & 108.16 \\
\hline DI2 & 61.8955 \\
\hline DI3 & 117.7863 \\
\hline DI4 & -119.6877 \\
\hline DI5 & -61.9315 \\
\hline DI6 & 119.7008 \\
\hline DI7 & -117.7801 \\
\hline DI8 & -125.9226 \\
\hline DI9 & 60.8493 \\
\hline DI10 & 129.8909 \\
\hline DI11 & -121.0429 \\
\hline \multicolumn{2}{|c|}{$B 3 L Y P /(D E L)$} \\
\hline \\
\hline \multicolumn{2}{|c|}{ C 1 PH1 } \\
\hline \multicolumn{2}{|c|}{ C 1 PH2 } \\
\hline
\end{tabular}


\begin{tabular}{lc} 
H 4 CH1 & 1 HCP \\
H 4 CH2 1 HCP2 \\
H 4 CH3 1 HCP3 \\
H 3 CH4 1 HCP \\
H 3 CH5 1 HCP5 \\
H 3 CH6 1 HCP6 \\
O 1 PO 2 OPH 3 \\
H 2 CH7 1 HCP7 \\
H 2 CH8 1 HCP8 \\
& \\
PH1 & 1.871567 \\
PH2 & 1.820800 \\
PC & 1.820956 \\
CH1 & 1.093572 \\
CH2 & 1.097763 \\
CH3 & 1.099172 \\
CH4 & 1.093660 \\
CH5 & 1.099104 \\
CH6 & 1.097750 \\
PO & 1.557018 \\
CH7 & 1.114060 \\
CH8 & 1.114111 \\
HPH & 108.398 \\
CPH & 108.580 \\
HCP & 107.043 \\
HCP2 & 109.553 \\
HCP3 & 113.059 \\
HCP4 & 106.980 \\
HCP5 & 113.029 \\
HCP6 & 109.641 \\
OPH & 117.794 \\
HCP7 & 102.001 \\
HCP8 & 102.078 \\
DI1 & 113.324 \\
DI2 & 53.619 \\
DI3 & 117.183 \\
DI4 & -119.561 \\
DI5 & -53.921 \\
DI6 & 119.442 \\
DI7 & -117.315 \\
DI8 & -123.235 \\
DI9 & 69.521 \\
DI10 & 107.341 \\
& \\
\hline
\end{tabular}

$\mathrm{C}_{2} \mathrm{H}_{4} \mathrm{PO}\left(\mathrm{CH}_{3}\right)_{2}^{-}$

B3LYP

$\mathrm{P}$

C 1 PH1

C 1 PH2 $2 \mathrm{HPH}$

C 1 PC 2 CPH 3 DI1

H 4 CH1 1 HCP 2 DI2

$\mathrm{H} 4 \mathrm{CH} 21 \mathrm{HCP} 25 \mathrm{DI} 3$

H 4 CH3 1 HCP3 5 DI4

H 3 CH4 1 HCP4 2 DI5

H 3 CH5 1 HCP5 8 DI6

H 3 CH6 1 HCP6 8 DI7

O 1 PO 2 OPH 3 DI8

H 2 CH7 1 HCP7 3 DI9

$\mathrm{X} 2 \mathrm{CH} 81$ HCP8 12 DI10

C 2 CH9 1 HCP9 12 DI1 1

H 14 CH10 2 HCC1 12 DI12

H 14 CH1 12 HCC2 15 DI13

H 14 CH12 2 HCC3 15 DI14

$\begin{array}{ll}\text { PH1 } & 1.7344 \\ \text { PH2 } & 1.8657 \\ \text { PC } & 1.8599 \\ \text { CH1 } & 1.0958 \\ \text { CH2 } & 1.0956 \\ \text { CH3 } & 1.0939 \\ \text { CH4 } & 1.0957 \\ \text { CH5 } & 1.0944 \\ \text { CH6 } & 1.0961 \\ \text { CH7 } & 1.0969 \\ \text { CH8 } & 1.0976 \mathrm{~F} \\ \text { CH9 } & 1.5223\end{array}$

$\begin{array}{lccc}\text { PO } & 1.542 & \text { OPH } & 125.7978 \\ \text { CH10 } & 1.1024 & \text { HCP7 } & 110.9307 \\ \text { CH11 } & 1.1015 & \text { HCP8 } & 108.8912 \\ \text { CH12 } & 1.1118 & \text { HCP9 } & 118.0309 \\ \text { HPH } & 107.0281 & \text { HCC1 } & 110.88 \\ \text { CPH } & 104.5327 & \text { HCC2 } & 110.954 \\ \text { HCP } & 108.3966 & \text { HCC3 } & 115.8517 \\ \text { HCP2 } & 108.9376 & \text { DI1 } & 108.2675 \\ \text { HCP3 } & 112.0832 & \text { DI2 } & 59.5961 \\ \text { HCP4 } & 108.5452 & \text { DI3 } & 117.7033 \\ \text { HCP5 } & 111.6808 & \text { DI4 } & -120.1828 \\ \text { HCP6 } & 109.5618 & \text { DI5 } & -62.422 \\ \text { OPH } & 126.0231 & \text { DI6 } & 119.9236 \\ \text { HCP7 } & 110.9775 & \text { DI7 } & -117.7018 \\ \text { HCP8 } & 108.8912 \text { F } & \text { DI8 } & -125.1916 \\ \text { HCP9 } & 119.6106 & \text { DI9 } & 202.7373 \\ \text { HCC1 } & 111.0381 & \text { DI10 } & 114.883 \\ \text { HCC2 } & 111.5458 & \text { DI11 } & -134.0676 \\ \text { HCC3 } & 116.2576 & \text { DI12 } & 60.0048 \\ \text { DI1 } & 108.3293 & \text { DI13 } & 117.3512 \\ \text { DI2 } & 58.3994 & \text { DI14 } & -121.6272\end{array}$

$\mathrm{C}_{3} \mathrm{H}_{4} \mathrm{PO}\left(\mathrm{CH}_{3}\right)_{2}^{-}$

$B 3 L Y P$

$\mathrm{C}$

C 1 CC

C $2 \mathrm{CC} 11 \mathrm{CCC}$

H 1 CH1 2 HCC1 3 DI 1

$\mathrm{H} 1 \mathrm{CH} 22 \mathrm{HCC} 24 \mathrm{DI} 2$

$\mathrm{H} 2 \mathrm{CH} 31 \mathrm{HCC} 34$ DI3

$\mathrm{H} 3 \mathrm{CH} 42 \mathrm{HCC} 46$ DI4

P 3 CH5 2 HCC5 7 DI5

C 8 PC 3 PCC 2 DI6

C 8 PC2 3 HCP 9 DI7

H 9 CH6 8 HCP2 10 DI8

H 9 CH7 8 HCP3 11 DI9

H 9 CH8 8 HCP4 11 DI10

H 10 CH9 8 HCP5 9 DI11

H 10 CH10 8 HCP6 14 DI12

H 10 CH11 8 HCP7 14 DI13

O 8 PO 3 OPC 9 DI14

$\begin{array}{lc}\text { CC } & 1.3777 \\ \text { CC1 } & 1.4171 \\ \text { CH1 } & 1.0888 \\ \text { CH2 } & 1.0866 \\ \text { CH3 } & 1.0974 \\ \text { CH4 } & 1.0888 \\ \text { CH5 } & 1.7402 \\ \text { PC } & 1.8594 \\ \text { PC2 } & 1.866 \\ \text { CH6 } & 1.0957 \\ \text { CH7 } & 1.0965 \\ \text { CH8 } & 1.0939 \\ \text { CH9 } & 1.0962 \\ \text { CH10 } & 1.0974 \\ \text { CH11 } & 1.0945 \\ \text { PO } & 1.5246 \\ \text { CCC } & 130.3771 \\ \text { HCC1 } & 121.7862 \\ \text { HCC2 } & 121.0285 \\ \text { HCC3 } & 114.2298 \\ \text { HCC4 } & 120.2379 \\ \text { HCC5 } & 125.458 \\ \text { PCC } & 108.7675 \\ \text { HCP } & 111.0034 \\ \text { HCP2 } & 111.8513 \\ \text { HCP3 } & 110.1877 \\ \text { HCP4 } & 107.6483 \\ \text { HCP5 } & 111.3206 \\ \text { HCP6 } & 110.8589 \\ \text { HCP7 } & 107.4957 \\ \text { OPC } & 116.3862 \\ \text { DI1 } & 0.5827 \\ \text { DI2 } & 180.562 \\ \text { DI3 } & 180.1967\end{array}$




\begin{tabular}{|c|c|c|c|}
\hline $\mathrm{PN}$ & 1.87 & \multicolumn{2}{|l|}{$B 3 L Y P$} \\
\hline NH1 & 1.0227 & \multicolumn{2}{|l|}{$\mathrm{C}$} \\
\hline NH2 & 1.0229 & \multicolumn{2}{|c|}{ C 1 CC } \\
\hline CH1 & $1.0988 \mathrm{~F}$ & \multicolumn{2}{|c|}{$\mathrm{C} 2 \mathrm{CC} 11 \mathrm{CCC}$} \\
\hline $\mathrm{CH} 2$ & 1.0927 & \multicolumn{2}{|c|}{ H 1 CH1 2 HCC1 3 DI1 } \\
\hline $\mathrm{CH} 3$ & 1.5125 & \multicolumn{2}{|c|}{ H 1 CH2 2 HCC2 4 DI2 } \\
\hline $\mathrm{CH} 4$ & 1.1119 & \multicolumn{2}{|c|}{ H 2 CH3 1 HCC 34 DI3 } \\
\hline $\mathrm{CH} 5$ & 1.1032 & \multicolumn{2}{|c|}{ H 3 CH4 2 HCC4 6 DI4 } \\
\hline CH6 & 1.1049 & \multicolumn{2}{|c|}{ P 3 CH5 2 HCC5 7 DI5 } \\
\hline $\mathrm{HPH}$ & 98.8178 & N 8 PN & 3 NPC 2 DI6 \\
\hline NPH & 113.1214 & $\mathrm{H} 8 \mathrm{PH}$ & 3 HPC 9 DI7 \\
\hline HNP1 & 109.2686 & $\mathrm{H} 9 \mathrm{NH}$ & 18 HNP 10 DI8 \\
\hline HNP2 & 114.4594 & $\mathrm{H} 9 \mathrm{NF}$ & 28 HNP2 10 DI9 \\
\hline HCP & $105.4603 \mathrm{~F}$ & & \\
\hline HCP2 & 114.6599 & $\mathrm{CC}$ & 1.3783 \\
\hline $\mathrm{HCP} 3$ & 126.2367 & $\mathrm{CC} 1$ & 1.419 \\
\hline $\mathrm{HCC} 1$ & 115.7236 & $\mathrm{CH} 1$ & 1.0891 \\
\hline $\mathrm{HCC} 2$ & 110.5929 & $\mathrm{CH} 2$ & 1.0871 \\
\hline $\mathrm{HCC} 3$ & 112.0314 & $\mathrm{CH} 3$ & 1.0974 \\
\hline DI1 & 98.1511 & $\mathrm{CH} 4$ & 1.0913 \\
\hline DI2 & -191.2146 & CH5 & 1.7581 \\
\hline DI3 & 120.2911 & PN & 1.8097 \\
\hline DI4 & $71.0203 \mathrm{~F}$ & $\mathrm{PH}$ & 1.4448 \\
\hline DI5 & 92.1509 & $\mathrm{NH} 1$ & 1.0215 \\
\hline DI6 & -108.2433 & $\mathrm{NH} 2$ & 1.0207 \\
\hline DI7 & 74.5589 & $\mathrm{CCC}$ & 130.2599 \\
\hline DI8 & 120.1283 & $\mathrm{HCC} 1$ & 121.8236 \\
\hline DI9 & -121.1836 & $\mathrm{HCC} 2$ & 121.1038 \\
\hline & & $\mathrm{HCC} 3$ & 115.2054 \\
\hline MP2 & & $\mathrm{HCC} 4$ & 118.065 \\
\hline $\mathrm{P}$ & & HCC5 & 126.3902 \\
\hline C 1 PH & & NPC & 107.4722 \\
\hline H 1 PH & I2 $2 \mathrm{HPH}$ & HPC & 97.7426 \\
\hline N 1 PN & 2 NPH 3 DI1 & HNP & 111.8641 \\
\hline $\mathrm{H} 4 \mathrm{Nl}$ & H1 1 HNP1 2 DI2 & HNP2 & 114.3339 \\
\hline $\mathrm{H} 4 \mathrm{Nl}$ & H2 1 HNP2 5 DI3 & DI1 & 2.0315 \\
\hline $\mathrm{X} 2 \mathrm{Cl}$ & I1 1 HCP 3 DI4 & DI2 & 180.5524 \\
\hline $\mathrm{H} 2 \mathrm{Cl}$ & 12 1 HCP2 7 DI5 & DI3 & 180.8735 \\
\hline $\mathrm{C} 2 \mathrm{CH}$ & I3 1 HCP3 7 DI6 & DI4 & 182.2722 \\
\hline $\mathrm{H} 9 \mathrm{Cl}$ & I4 2 HCC1 8 DI7 & DI5 & 180.2043 \\
\hline $\mathrm{H} 9 \mathrm{Cl}$ & 152 HCC2 10 DI8 & DI6 & 80.3031 \\
\hline $\mathrm{H} 9 \mathrm{Cl}$ & $\mathrm{H} 62$ HCC 310 DI9 & DI7 & -101.5545 \\
\hline & & DI8 & -76.7049 \\
\hline PH & 1.7224 & DI9 & 47.9653 \\
\hline $\mathrm{PH} 2$ & 1.4311 & & \\
\hline PN & 1.8582 & $M P 2$ & \\
\hline NH1 & 1.0206 & $\mathrm{C}$ & \\
\hline NH2 & 1.0205 & $\mathrm{C} 1 \mathrm{CC}$ & \\
\hline $\mathrm{CH} 1$ & 1.0988 & $\mathrm{C} 2 \mathrm{CC}$ & $11 \mathrm{CCC}$ \\
\hline $\mathrm{CH} 2$ & 1.0882 & $\mathrm{H} 1 \mathrm{CH}$ & 12 HCC1 3 DI1 \\
\hline $\mathrm{CH} 3$ & 1.51 & $\mathrm{H} 1 \mathrm{CH}$ & 22 HCC2 4 DI2 \\
\hline $\mathrm{CH} 4$ & 1.1037 & $\mathrm{H} 2 \mathrm{CH}$ & 31 HCC3 4 DI3 \\
\hline CH5 & 1.0979 & $\mathrm{H} 3 \mathrm{CH}$ & 42 HCC4 6 DI4 \\
\hline CH6 & 1.0981 & $\mathrm{P} 3 \mathrm{CH}$ & 52 HCC5 7 DI5 \\
\hline $\mathrm{HPH}$ & 98.2891 & N 8 PN & 3 NPC 2 DI6 \\
\hline NPH & 112.5917 & $\mathrm{H} 8 \mathrm{PH}$ & 3 HPC 9 DI7 \\
\hline HNP1 & 109.5618 & $\mathrm{H} 9 \mathrm{NH}$ & 18 HNP 10 DI8 \\
\hline HNP2 & 114.0853 & $\mathrm{H} 9 \mathrm{NH}$ & 28 HNP2 10 DI9 \\
\hline HCP & 105.4603 & & \\
\hline HCP2 & 115.2307 & $\mathrm{CC}$ & 1.3771 \\
\hline $\mathrm{HCP} 3$ & 124.3106 & $\mathrm{CC} 1$ & 1.421 \\
\hline $\mathrm{HCC} 1$ & 115.5891 & $\mathrm{CH} 1$ & 1.0848 \\
\hline $\mathrm{HCC} 2$ & 109.9049 & $\mathrm{CH} 2$ & 1.0829 \\
\hline $\mathrm{HCC} 3$ & 111.8812 & $\mathrm{CH} 3$ & 1.0947 \\
\hline DI1 & 97.9258 & $\mathrm{CH} 4$ & 1.0873 \\
\hline DI2 & -188.5428 & CH5 & 1.7486 \\
\hline DI3 & 120.4694 & PN & 1.8008 \\
\hline DI4 & 71.0203 & $\mathrm{PH}$ & 1.427 \\
\hline DI5 & 93.1709 & $\mathrm{NH} 1$ & 1.0193 \\
\hline DI6 & -110.1435 & $\mathrm{NH} 2$ & 1.0183 \\
\hline DI7 & 74.2939 & $\mathrm{CCC}$ & 129.9978 \\
\hline DI8 & 119.9645 & $\mathrm{HCC} 1$ & 121.6237 \\
\hline DI9 & -121.6789 & $\mathrm{HCC} 2$ & 120.9831 \\
\hline & & $\mathrm{HCC} 3$ & 115.3431 \\
\hline & & $\mathrm{HCC} 4$ & 118.351 \\
\hline $\mathrm{C}_{3} \mathrm{H}_{4} \mathrm{P}$ & $\mathrm{HNH}_{2}^{-}$ & HCC5 & 124.8303 \\
\hline & & NPC & 107.1794 \\
\hline
\end{tabular}

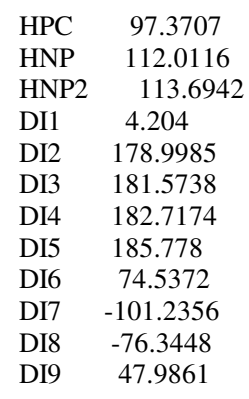

$\mathrm{CH}_{3} \mathrm{P}\left(\mathrm{NH}_{2}\right)_{2}$

B3LYP

$\mathrm{P}$

$\mathrm{C} 1 \mathrm{PH}$

N 1 PH2 2 HPH

N 1 PN 2 NPH 3 DI1

H 4 NH1 1 HNP1 2 DI2

$\mathrm{H} 4 \mathrm{NH} 21$ HNP2 5 DI3

H 3 NH3 1 HNP3 2 DI4

H 3 NH4 1 HNP4 7 DI5

H 2 CH1 1 HCP 3 DI6

$\mathrm{H} 2 \mathrm{CH} 21$ HCP2 9 DI7

$\mathrm{H} 2 \mathrm{CH} 31 \mathrm{HCP} 39$ DI8

\begin{tabular}{|c|c|}
\hline $\mathrm{PH}$ & 1.8525 \\
\hline PH2 & 1.731 \\
\hline PN & 1.7307 \\
\hline NH1 & 1.0166 \\
\hline $\mathrm{NH} 2$ & 1.0125 \\
\hline NH3 & 1.0167 \\
\hline NH4 & 1.0125 \\
\hline $\mathrm{CH} 1$ & 1.0928 \\
\hline $\mathrm{CH} 2$ & 1.0929 \\
\hline $\mathrm{CH} 3$ & 1.0987 \\
\hline HPH & 96.9168 \\
\hline NPH & 96.9015 \\
\hline HNP1 & 120.3975 \\
\hline HNP2 & 115.783 \\
\hline HNP3 & 120.3816 \\
\hline HNP4 & 115.7378 \\
\hline HCP & 109.3206 \\
\hline $\mathrm{HCP} 2$ & 109.323 \\
\hline $\mathrm{HCP} 3$ & 111.9373 \\
\hline DI1 & 111.6854 \\
\hline DI2 & -52.1529 \\
\hline DI3 & 221.193 \\
\hline DI4 & 52.1935 \\
\hline DI5 & 138.6703 \\
\hline DI6 & 63.5452 \\
\hline DI7 & 119.416 \\
\hline DI8 & -120.3296 \\
\hline$M P 2$ & \\
\hline $\begin{array}{l}\mathrm{P} \\
\mathrm{C} 1\end{array}$ & \\
\hline
\end{tabular}

N 1 PH2 2 HPH

N 1 PN 2 NPH 3 DI1

H 4 NH1 1 HNP1 2 DI2

$\mathrm{H} 4 \mathrm{NH} 21$ HNP2 5 DI3

H 3 NH3 1 HNP3 2 DI4

H 3 NH4 1 HNP4 7 DI5

H 2 CH1 1 HCP 3 DI6

$\mathrm{H} 2 \mathrm{CH} 21 \mathrm{HCP} 29$ DI7

H 2 CH3 1 HCP3 9 DI8

$\begin{array}{lc}\mathrm{PH} & 1.8358 \\ \mathrm{PH} 2 & 1.7248 \\ \mathrm{PN} & 1.7247 \\ \mathrm{NH} 1 & 1.0143 \\ \mathrm{NH} 2 & 1.0105 \\ \mathrm{NH} 3 & 1.0143 \\ \mathrm{NH} 4 & 1.0105 \\ \mathrm{CH} 1 & 1.0881\end{array}$




$\begin{array}{lc}\mathrm{CH} 2 & 1.0882 \\ \mathrm{CH} 3 & 1.0938 \\ \mathrm{HPH} & 96.4004 \\ \mathrm{NPH} & 96.4088 \\ \mathrm{HNP} 1 & 119.6353 \\ \mathrm{HNP} 2 & 115.8804 \\ \mathrm{HNP3} & 119.6023 \\ \mathrm{HNP} 4 & 115.8469 \\ \mathrm{HCP} & 109.5127 \\ \mathrm{HCP} 2 & 109.5163 \\ \mathrm{HCP} 3 & 111.2281 \\ \mathrm{DI} 1 & 111.6216 \\ \mathrm{DI} 2 & -52.4921 \\ \text { DI3 } & 222.0971 \\ \text { DI4 } & 52.5484 \\ \text { DI5 } & 137.7731 \\ \text { DI6 } & 64.3087 \\ \text { DI7 } & 119.7302 \\ \text { DI8 } & -120.1398\end{array}$

$\mathrm{CH}_{2} \mathrm{P}\left(\mathrm{NH}_{2}\right)_{2}^{-}$

B3LYP
P
N 1 PN1
N 1 PN2 2 NPN
H 2 NH1 1 HNP1 3 DI1
H 2 NH2 1 HNP2 4 DI2
H 3 NH3 1 HNP3 2 DI3
H 3 NH4 1 HNP4 6 DI4
C 1 PC1 2 CPN 3 DI5
H 8 CH1 1 HCP1 2 DI6
H 8 CH2 1 HCP2 9 DI7

$\begin{array}{lc}\text { PN1 } & 1.8151 \\ \text { PN2 } & 1.8282 \\ \text { NH1 } & 1.0198 \\ \text { NH2 } & 1.0168 \\ \text { NH3 } & 1.0248 \\ \text { NH4 } & 1.0197 \\ \text { PC1 } & 1.7249 \\ \text { CH1 } & 1.0879 \\ \text { CH2 } & 1.0937 \\ \text { NPN } & 88.148 \\ \text { HNP1 } & 110.7202 \\ \text { HNP2 } & 107.4724 \\ \text { HNP3 } & 109.0142 \\ \text { HNP4 } & 106.7301 \\ \text { CPN } & 101.3232 \\ \text { HCP1 } & 117.6104 \\ \text { HCP2 } & 124.1182 \\ \text { DI1 } & 293.5656 \\ \text { DI2 } & -118.321 \\ \text { DI3 } & -57.9188 \\ \text { DI4 } & -114.9704 \\ \text { DI5 } & -113.8292 \\ \text { DI6 } & -147.2062 \\ \text { DI7 } & -169.0903 \\ & \end{array}$

$M P 2$

$\mathrm{P}$

N 1 PN1

N 1 PN2 2 NPN

H 2 NH1 1 HNP1 3 DI1

H 2 NH2 1 HNP2 4 DI2

H 3 NH3 1 HNP3 2 DI3

H 3 NH4 1 HNP4 6 DI4

C 1 PC1 2 CPN 3 DI5

H 8 CH1 1 HCP1 2 DI6

$\mathrm{H} 8 \mathrm{CH} 21 \mathrm{HCP} 29 \mathrm{DI} 7$

$\begin{array}{ll}\text { PN1 } & 1.8038 \\ \text { PN2 } & 1.8154 \\ \text { NH1 } & 1.0184 \\ \text { NH2 } & 1.0155 \\ \text { NH3 } & 1.023 \\ \text { NH4 } & 1.018\end{array}$

$\begin{array}{lc}\text { PC1 } & 1.718 \\ \text { CH1 } & 1.0839 \\ \text { CH2 } & 1.0894 \\ \text { NPN } & 87.4411 \\ \text { HNP1 } & 110.6797 \\ \text { HNP2 } & 107.6721 \\ \text { HNP3 } & 108.4275 \\ \text { HNP4 } & 107.0265 \\ \text { CPN } & 100.6213 \\ \text { HCP1 } & 117.7067 \\ \text { HCP2 } & 123.5024 \\ \text { DI1 } & 295.5093 \\ \text { DI2 } & -118.4324 \\ \text { DI3 } & -56.6202 \\ \text { DI4 } & -115.0968 \\ \text { DI5 } & -113.839 \\ \text { DI6 } & -147.5931 \\ \text { DI7 } & -169.0669\end{array}$

\section{$B 3 L Y P /(D E L)$}

$\mathrm{P}$

C $1 \mathrm{PH}$

N 1 PH2 2 HPH

N 1 PN 2 NPH 3 DI 1

H 4 NH1 1 HNP1 2 DI2

H 4 NH2 1 HNP2 5 DI3

$\mathrm{H} 3 \mathrm{NH} 31 \mathrm{HNP} 32$ DI4

H 3 NH4 1 HNP4 7 DI5

H 2 CH1 1 HCP 3 DI6

H 2 CH3 1 HCP3 9 DI8

$\begin{array}{lc}\text { PH } & 1.8507 \\ \text { PH2 } & 1.7536 \\ \text { PN } & 1.7537 \\ \text { NH1 } & 1.0276 \\ \text { NH2 } & 1.012 \\ \text { NH3 } & 1.0276 \\ \text { NH4 } & 1.012 \\ \text { CH1 } & 1.1059 \\ \text { CH3 } & 1.1059 \\ \text { HPH } & 96.2485 \\ \text { NPH } & 96.2435 \\ \text { HNP1 } & 111.4978 \\ \text { HNP2 } & 115.0658 \\ \text { HNP3 } & 111.452 \\ \text { HNP4 } & 115.0022 \\ \text { HCP } & 107.246 \\ \text { HCP3 } & 107.2635 \\ \text { DI1 } & 109.6169 \\ \text { DI2 } & -34.9031 \\ \text { DI3 } & 232.1232 \\ \text { DI4 } & 35.0115 \\ \text { DI5 } & 127.7703 \\ \text { DI6 } & 68.4178 \\ \text { DI8 } & -246.5223 \\ & \end{array}$

$\mathrm{C}_{2} \mathrm{H}_{4} \mathrm{P}\left(\mathrm{NH}_{2}\right)_{2}{ }^{-}$

B3LYP

$\mathrm{P}$

C $1 \mathrm{PH}$

N 1 PH2 2 HPH

N 1 PN 2 NPH 3 DI 1

H 4 NH1 1 HNP1 2 DI2

H 4 NH2 1 HNP2 5 DI3

$\mathrm{H} 3 \mathrm{NH} 31 \mathrm{HNP} 32 \mathrm{DI} 4$

H 3 NH4 1 HNP4 7 DI5

H 2 CH1 1 HCP 3 DI6

$\mathrm{X} 2 \mathrm{CH} 21 \mathrm{HCP} 29 \mathrm{DI} 7$

C 2 CH3 1 HCP3 9 DI8

H 11 CH4 2 HCC1 10 DI9

H 11 CH5 2 HCC2 12 DI10

H 11 CH6 2 HCC3 12 DI11

$\begin{array}{ll}\text { PH } & 1.726 \\ \text { PH2 } & 1.8726 \\ \text { PN } & 1.7967\end{array}$ \begin{tabular}{lc} 
NH1 & 1.0172 \\
NH2 & 1.0173 \\
NH3 & 1.0208 \\
NH4 & 1.0255 \\
CH1 & 1.0987 \\
CH2 & $1.0954 \mathrm{~F}$ \\
CH3 & 1.5122 \\
CH4 & 1.1008 \\
CH5 & 1.1071 \\
CH6 & 1.1105 \\
HPH & 113.5238 \\
NPH & 102.2709 \\
HNP1 & 110.154 \\
HNP2 & 111.1742 \\
HNP3 & 106.6969 \\
HNP4 & 108.5307 \\
HCP & 120.0483 \\
HCP2 & $106.0543 \mathrm{~F}$ \\
HCP3 & 122.2019 \\
HCC1 & 110.2019 \\
HCC2 & 113.1397 \\
HCC3 & 115.2347 \\
DI1 & 96.6285 \\
DI2 & 3.1491 \\
DI3 & 237.2717 \\
DI4 & -94.8769 \\
DI5 & 113.4055 \\
DI6 & -56.8622 \\
DI7 & $114.5224 \mathrm{~F}$ \\
DI8 & -191.8249 \\
DI9 & 99.0857 \\
DI10 & 118.7624 \\
DI11 & -119.738 \\
& \\
\hline
\end{tabular}

$M P 2$

$\mathrm{P}$

C $1 \mathrm{PH}$

N 1 PH2 $2 \mathrm{HPH}$

N 1 PN 2 NPH 3 DI1

H 4 NH1 1 HNP1 2 DI2

$\mathrm{H} 4 \mathrm{NH} 21 \mathrm{HNP} 25 \mathrm{DI} 3$

H 3 NH3 1 HNP3 2 DI4

H 3 NH4 1 HNP4 7 DI5

H 2 CH1 1 HCP 3 DI6

$\mathrm{X} 2 \mathrm{CH} 21 \mathrm{HCP} 29 \mathrm{DI} 7$

C 2 CH3 1 HCP3 9 DI8

$\mathrm{H} 11 \mathrm{CH} 42$ HCC1 10 DI9

H 11 CH5 2 HCC2 12 DI10

H 11 CH6 2 HCC3 12 DI11

$\begin{array}{lc}\text { PH } & 1.7197 \\ \text { PH2 } & 1.8557 \\ \text { PN } & 1.7862 \\ \text { NH1 } & 1.015 \\ \text { NH2 } & 1.0151 \\ \text { NH3 } & 1.0188 \\ \text { NH4 } & 1.0241 \\ \text { CH1 } & 1.0943 \\ \text { CH2 } & 1.0954 \\ \text { CH3 } & 1.5097 \\ \text { CH4 } & 1.0956 \\ \text { CH5 } & 1.0993 \\ \text { CH6 } & 1.1019 \\ \text { HPH } & 114.5628 \\ \text { NPH } & 101.529 \\ \text { HNP1 } & 110.4012 \\ \text { HNP2 } & 112.1752 \\ \text { HNP3 } & 106.9758 \\ \text { HNP4 } & 108.524 \\ \text { HCP } & 119.9666 \\ \text { HCP2 } & 106.0543 \\ \text { HCP3 } & 121.6092 \\ \text { HCC1 } & 109.5541 \\ \text { HCC2 } & 112.6982 \\ \text { HCC3 } & 115.1802 \\ \text { DI1 } & 95.6029 \\ \text { DI2 } & 5.8624 \\ & \end{array}$




$\begin{array}{ll}\text { DI3 } & 235.7414 \\ \text { DI4 } & -92.7647 \\ \text { DI5 } & 113.9667 \\ \text { DI6 } & -56.215 \\ \text { DI7 } & 114.5224 \\ \text { DI8 } & -193.1742 \\ \text { DI9 } & 99.9037 \\ \text { DI10 } & 118.5344 \\ \text { DI11 } & -119.6176\end{array}$

$\mathrm{C}_{3} \mathrm{H}_{4} \mathrm{P}\left(\mathrm{NH}_{2}\right)_{2}^{-}$

B3LYP

$\mathrm{C}$

$\mathrm{C} 1 \mathrm{CC}$

$\mathrm{C} 2 \mathrm{CC} 11 \mathrm{CCC}$

H 1 CH1 2 HCC1 3 DI1

$\mathrm{H} 1 \mathrm{CH} 22 \mathrm{HCC} 24$ DI2

$\mathrm{H} 2 \mathrm{CH} 31 \mathrm{HCC} 34 \mathrm{DI} 3$

$\mathrm{H} 3 \mathrm{CH} 42 \mathrm{HCC} 46$ DI4

P 3 CH5 2 HCC5 7 DI5

N 8 PN 3 NPC 2 DI6

$\mathrm{N} 8 \mathrm{PH} 3 \mathrm{HPC} 9 \mathrm{DI} 7$

H 9 NH1 8 HNP 10 DI8

H 9 NH2 8 HNP2 10 DI9

H 10 NH3 8 HNP3 9 DI10

H 10 NH4 8 HNP4 13 DI11

$\begin{array}{ll}\text { CC } & 1.3771 \\ \text { CC1 } & 1.4188 \\ \text { CH1 } & 1.0894 \\ \text { CH2 } & 1.0869 \\ \text { CH3 } & 1.0962 \\ \text { CH4 } & 1.098 \\ \text { CH5 } & 1.7535 \\ \text { PN } & 1.7828 \\ \text { PH } & 1.8254 \\ \text { NH1 } & 1.0178 \\ \text { NH2 } & 1.0185 \\ \text { NH3 } & 1.0199 \\ \text { NH4 } & 1.024 \\ \text { CCC } & 130.1804 \\ \text { HCC1 } & 121.8822 \\ \text { HCC2 } & 121.0627 \\ \text { HCC3 } & 115.3926 \\ \text { HCC4 } & 118.2767 \\ \text { HCC5 } & 122.5034 \\ \text { NPC } & 101.4353 \\ \text { HPC } & 109.0303 \\ \text { HNP } & 111.2389 \\ \text { HNP2 } & 111.9067 \\ \text { HNP3 } & 107.7389 \\ \text { HNP4 } & 110.5384 \\ \text { DI1 } & 0.0914 \\ \text { DI2 } & 180.9756 \\ \text { DI3 } & 179.9082 \\ \text { DI4 } & 180.4582 \\ \text { DI5 } & 182.565 \\ \text { DI6 } & 219.8791 \\ \text { DI7 } & -99.1247 \\ \text { DI8 } & -26.9498 \\ \text { DI9 } & 95.7602 \\ \text { DI10 } & 164.2469 \\ \text { DI11 } & 115.5614 \\ & \end{array}$

$M P 2$

$\mathrm{C}$

C 1 CC

C 2 CC $11 \mathrm{CCC}$

H 1 CH1 2 HCC1 3 DI 1

$\mathrm{H} 1 \mathrm{CH} 22 \mathrm{HCC} 24$ DI2

$\mathrm{H} 2 \mathrm{CH} 31 \mathrm{HCC} 34 \mathrm{DI} 3$

$\mathrm{H} 3 \mathrm{CH} 42$ HCC4 6 DI4

P 3 CH5 2 HCC5 7 DI5

N 8 PN 3 NPC 2 DI6

N 8 PH 3 HPC 9 DI7

H 9 NH1 8 HNP 10 DI8
H 9 NH2 8 HNP2 10 DI9 H 10 NH3 8 HNP3 9 DI10 H 10 NH4 8 HNP4 13 DI1 1

$\begin{array}{lc}\text { CC } & 1.3753 \\ \text { CC1 } & 1.4212 \\ \text { CH1 } & 1.0849 \\ \text { CH2 } & 1.0827 \\ \text { CH3 } & 1.0928 \\ \text { CH4 } & 1.0942 \\ \text { CH5 } & 1.7426 \\ \text { PN } & 1.7748 \\ \text { PH } & 1.8119 \\ \text { NH1 } & 1.0156 \\ \text { NH2 } & 1.0157 \\ \text { NH3 } & 1.018 \\ \text { NH4 } & 1.0222 \\ \text { CCC } & 129.6723 \\ \text { HCC1 } & 121.682 \\ \text { HCC2 } & 120.9506 \\ \text { HCC3 } & 115.6953 \\ \text { HCC4 } & 118.5051 \\ \text { HCC5 } & 121.5186 \\ \text { NPC } & 100.9 \\ \text { HPC } & 109.9549 \\ \text { HNP } & 11.7878 \\ \text { HNP2 } & 111.9147 \\ \text { HNP3 } & 108.077 \\ \text { HNP4 } & 110.472 \\ \text { DI1 } & 0.7685 \\ \text { DI2 } & 181.0686 \\ \text { DI3 } & 180.0261 \\ \text { DI4 } & 180.3772 \\ \text { DI5 } & 185.7042 \\ \text { DI6 } & 216.9415 \\ \text { DI7 } & -98.1105 \\ \text { DI8 } & -23.3121 \\ \text { DI9 } & 100.6484 \\ \text { DI10 } & 167.8646 \\ \text { DI11 } & 116.1583\end{array}$

$\mathrm{CH}_{3} \mathrm{PO}\left(\mathrm{NH}_{2}\right)_{2}$

B3LYP

$\mathrm{P}$

$\mathrm{C} 1 \mathrm{PH}$

N 1 PH2 $2 \mathrm{HPH}$

N 1 PN 2 NPH 3 DI1

H 4 NH1 1 HNP1 2 DI2

H 4 NH2 1 HNP2 5 DI3

H 3 NH3 1 HNP3 2 DI4

H 3 NH4 1 HNP4 7 DI5

O 1 PO 2 OPH 3 DI6

H 2 CH1 1 HCP1 3 DI7

$\mathrm{H} 2 \mathrm{CH} 21 \mathrm{HCP} 210 \mathrm{DI} 8$

H 2 CH3 1 HCP3 10 DI9

$\begin{array}{lc}\text { PH } & 1.8269 \\ \text { PH2 } & 1.6856 \\ \text { PN } & 1.6983 \\ \text { NH1 } & 1.0155 \\ \text { NH2 } & 1.0162 \\ \text { NH3 } & 1.012 \\ \text { NH4 } & 1.0117 \\ \text { PO } & 1.496 \\ \text { CH1 } & 1.093 \\ \text { CH2 } & 1.093 \\ \text { CH3 } & 1.0946 \\ \text { HPH } & 110.8868 \\ \text { NPH } & 101.7171 \\ \text { HNP1 } & 114.5415 \\ \text { HNP2 } & 112.8639 \\ \text { HNP3 } & 120.0577 \\ \text { HNP4 } & 115.344 \\ \text { OPH } & 112.5837 \\ \text { HCP1 } & 111.7183 \\ \text { HCP2 } & 108.3093\end{array}$

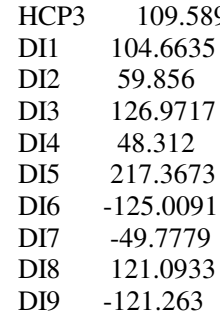

$M P 2$

$\mathrm{P}$

$\mathrm{C} 1 \mathrm{PH}$

N 1 PH2 2 HPH

N 1 PN 2 NPH 3 DI1

H 4 NH1 1 HNP1 2 DI2

$\mathrm{H} 4 \mathrm{NH} 21 \mathrm{HNP} 25$ DI3

H 3 NH3 1 HNP3 2 DI4

H 3 NH4 1 HNP4 7 DI5

O 1 PO 2 OPH 3 DI6

H 2 CH1 1 HCP1 3 DI7

$\mathrm{H} 2 \mathrm{CH} 21 \mathrm{HCP} 210 \mathrm{DI} 8$

H 2 CH3 1 HCP3 10 DI9

PH 1.8132

PH2 1.6817

PN $\quad 1.692$

NH1 1.0137

$\mathrm{NH} 2 \quad 1.0144$

$\mathrm{NH} 3 \quad 1.0104$

$\mathrm{NH} 4 \quad 1.0103$

PO 1.5009

CH1 1.0885

CH2 1.0884

$\mathrm{CH} 3 \quad 1.09$

HPH 111.0574

NPH $\quad 101.2776$

HNP1 114.4098

HNP2 112.7264

HNP3 119.044

HNP4 115.0564

OPH 112.6111

HCP1 111.3543

HCP2 108.5923

HCP3 109.4854

DI1 103.7521

DI2 60.2001

DI3 126.6178

DI4 47.8537

DI5 220.2317

DI6 -125.485

DI7 -49.8861

DI8 121.1308

DI9 -121.0272

$\mathrm{CH}_{2} \mathrm{PO}\left(\mathrm{NH}_{2}\right)_{2}^{-}$

$B 3 L Y P$

$\mathrm{P}$

O 1 PO

N 1 PN1 2 NPO1

N 1 PN2 2 NPO2 3 DI

H 3 NH1 1 HNP1 2 DI2

H 3 NH2 1 HNP2 5 DI3

H 4 NH3 1 HNP3 2 DI4

H 4 NH4 1 HNP4 7 DI5

C 1 PC1 2 OPC1 3 DI6

H 9 CH1 1 HCP1 2 DI7

H 9 CH2 1 HCP2 10 DI8

$\begin{array}{ll}\text { PO } & 1.523 \\ \text { PN1 } & 1.7365 \\ \text { PN2 } & 1.7835 \\ \text { NH1 } & 1.0149 \\ \text { NH2 } & 1.0165\end{array}$ 


\begin{tabular}{|c|c|c|c|c|c|}
\hline NH3 & 1.0178 & $\mathrm{PH}$ & 1.88 & DI4 & 19.0983 \\
\hline NH4 & 1.0178 & $\mathrm{PH} 2$ & 1.7394 & DI5 & 242.1766 \\
\hline PC1 & 1.7078 & $\mathrm{PN}$ & 1.7133 & DI6 & -126.0971 \\
\hline $\mathrm{CH} 1$ & 1.0869 & NH1 & 1.0163 & DI7 & 297.4418 \\
\hline $\mathrm{CH} 2$ & 1.0883 & $\mathrm{NH} 2$ & 1.0227 & DI8 & $114.941 \mathrm{~F}$ \\
\hline NPO1 & 116.7686 & NH3 & 1.0183 & DI9 & -203.065 \\
\hline NPO2 & 103.2373 & $\mathrm{NH} 4$ & 1.0178 & DI10 & 62.7603 \\
\hline HNP1 & 110.2933 & $\mathrm{PO}$ & 1.5066 & DI11 & 120.6314 \\
\hline HNP2 & 111.2619 & $\mathrm{CH} 1$ & 1.1051 & DI12 & -121.1638 \\
\hline HNP3 & 106.7627 & $\mathrm{CH} 2$ & 1.1039 & & \\
\hline HNP4 & 110.4515 & $\mathrm{HPH}$ & 104.5761 & $M P 2$ & \\
\hline OPC1 & 117.9149 & $\mathrm{NPH}$ & 113.2449 & $\mathrm{P}$ & \\
\hline HCP1 & 117.1074 & HNP1 & 110.1532 & $\mathrm{C} 1 \mathrm{PH}$ & \\
\hline HCP2 & 119.205 & HNP2 & 113.9058 & N $1 \mathrm{PH}$ & $\mathrm{H} 22 \mathrm{HPH}$ \\
\hline DI1 & 101.2051 & HNP3 & 106.7071 & N 1 PN & N 2 NPH 3 DI1 \\
\hline DI2 & -69.7583 & HNP4 & 109.7545 & $\mathrm{H} 4 \mathrm{NF}$ & H1 1 HNP1 2 DI 2 \\
\hline DI3 & 123.2513 & $\mathrm{OPH}$ & 116.3445 & $\mathrm{H} 4 \mathrm{NH}$ & $\mathrm{H} 21 \mathrm{HNP} 25 \mathrm{DI} 3$ \\
\hline DI4 & 37.3133 & HCP1 & 107.2221 & $\mathrm{H} 3 \mathrm{NH}$ & H3 1 HNP3 2 DI4 \\
\hline DI5 & 116.9986 & HCP2 & 108.3356 & H $3 \mathrm{NF}$ & H4 1 HNP4 7 DI5 \\
\hline DI6 & -124.5728 & DI1 & 105.0916 & $\mathrm{O} 1 \mathrm{PC}$ & 2 OPH 3 DI6 \\
\hline DI7 & -31.9231 & DI2 & 47.9983 & $\mathrm{H} 2 \mathrm{Cr}$ & H1 1 HCP1 3 DI7 \\
\hline DI8 & -151.4416 & DI3 & 123.8409 & $\mathrm{X} 2 \mathrm{CH}$ & H2 1 HCP2 10 DI8 \\
\hline & & DI4 & -64.105 & $\mathrm{C} 2 \mathrm{CH}$ & H3 1 HCP3 10 DI9 \\
\hline$M P 2$ & & DI5 & 115.5895 & $\mathrm{H} 12 \mathrm{C}$ & CH4 2 HCC1 10 DI10 \\
\hline $\mathrm{P}$ & & DI6 & -120.0322 & $\mathrm{H} 12 \mathrm{C}$ & CH5 2 HCC2 13 DI1 1 \\
\hline $\mathrm{O} 1 \mathrm{PC}$ & & DI7 & -65.9651 & $\mathrm{H} 12 \mathrm{C}$ & CH6 2 HCC3 13 DI12 \\
\hline N 1 PI & I 12 NPO1 & DI8 & 242.8908 & & \\
\hline N 1 PI & I2 2 NPO2 3 DI1 & & & $\mathrm{PH}$ & 1.7013 \\
\hline H $3 \mathrm{~N}$ & H1 1 HNP1 2 DI 2 & $\mathrm{C}_{2} \mathrm{H}_{4} \mathrm{P}$ & $\mathrm{O}\left(\mathrm{NH}_{2}\right)_{2}^{-}$ & $\mathrm{PH} 2$ & 1.7787 \\
\hline $\mathrm{H} 3 \mathrm{~N}$ & H2 1 HNP2 5 DI3 & & & PN & 1.7252 \\
\hline H $4 \mathrm{~N}$ & H3 1 HNP3 2 DI4 & $B 3 L Y P$ & & NH1 & 1.0129 \\
\hline $\mathrm{H} 4 \mathrm{~N}$ & H4 1 HNP4 7 DI5 & $\mathrm{P}$ & & $\mathrm{NH} 2$ & 1.0145 \\
\hline C 1 PC & 12 OPC1 3 DI6 & $\mathrm{C} 1 \mathrm{PH}$ & & NH3 & 1.0162 \\
\hline $\mathrm{H} 9 \mathrm{Cl}$ & $\mathrm{H} 11 \mathrm{HCP} 12 \mathrm{DI} 7$ & $\mathrm{~N} 1 \mathrm{PH}$ & $122 \mathrm{HPH}$ & NH4 & 1.0159 \\
\hline $\mathrm{H} 9 \mathrm{Cl}$ & 12 1 HCP2 10 DI 8 & N 1 PN & N 2 NPH 3 DI1 & $\mathrm{PO}$ & 1.53 \\
\hline & & $\mathrm{H} 4 \mathrm{NH}$ & H1 1 HNP1 2 DI2 & $\mathrm{CH} 1$ & 1.0877 \\
\hline $\mathrm{PO}$ & 1.5272 & $\mathrm{H} 4 \mathrm{NH}$ & H2 1 HNP2 5 DI3 & $\mathrm{CH} 2$ & 1.096 \\
\hline PN1 & 1.7274 & $\mathrm{H} 3 \mathrm{NH}$ & H3 1 HNP3 2 DI4 & $\mathrm{CH} 3$ & 1.5114 \\
\hline PN2 & 1.7716 & $\mathrm{H} 3 \mathrm{NI}$ & H4 1 HNP4 7 DI5 & $\mathrm{CH} 4$ & 1.1043 \\
\hline NH1 & 1.0132 & $\mathrm{O} 1 \mathrm{PC}$ & 2 OPH 3 DI6 & CH5 & 1.093 \\
\hline $\mathrm{NH} 2$ & 1.0146 & $\mathrm{H} 2 \mathrm{CH}$ & H1 1 HCP1 3 DI7 & CH6 & 1.0971 \\
\hline NH3 & 1.0161 & $\mathrm{X} 2 \mathrm{CH}$ & I2 1 HCP2 10 DI8 & $\mathrm{HPH}$ & 120.8138 \\
\hline NH4 & 1.0161 & $\mathrm{C} 2 \mathrm{CH}$ & $\mathrm{H} 31 \mathrm{HCP} 310 \mathrm{DI} 9$ & NPH & 103.1798 \\
\hline $\mathrm{PC} 1$ & 1.705 & $\mathrm{H} 12 \mathrm{C}$ & H4 2 HCC1 10 DI10 & HNP1 & 110.6702 \\
\hline $\mathrm{CH} 1$ & 1.084 & $\mathrm{H} 12 \mathrm{C}$ & H5 2 HCC2 13 DI11 & HNP2 & 111.0441 \\
\hline $\mathrm{CH} 2$ & 1.0855 & $\mathrm{H} 12 \mathrm{C}$ & H6 2 HCC3 13 DI12 & HNP3 & 110.927 \\
\hline NPO1 & 117.6449 & & & HNP4 & 107.5061 \\
\hline $\mathrm{NPO} 2$ & 103.1666 & $\mathrm{PH}$ & 1.7051 & $\mathrm{OPH}$ & 117.3286 \\
\hline HNP1 & 110.382 & PH2 & 1.7908 & HCP1 & 115.3216 \\
\hline HNP2 & 111.2148 & $\mathrm{PN}$ & 1.7341 & $\mathrm{HCP} 2$ & 108.5752 \\
\hline HNP3 & 107.1159 & NH1 & 1.0146 & $\mathrm{HCP} 3$ & 120.1128 \\
\hline HNP4 & 110.5993 & $\mathrm{NH} 2$ & 1.0162 & $\mathrm{HCC} 1$ & 115.5869 \\
\hline $\mathrm{OPC} 1$ & 117.7848 & NH3 & 1.0177 & $\mathrm{HCC} 2$ & 109.5655 \\
\hline HCP1 & 116.3361 & NH4 & 1.0175 & HCC 3 & 111.7245 \\
\hline $\mathrm{HCP} 2$ & 117.4887 & $\mathrm{PO}$ & 1.5254 & DI1 & 101.9595 \\
\hline DI1 & 100.8566 & $\mathrm{CH} 1$ & 1.0906 & DI2 & 62.4309 \\
\hline DI2 & -71.1584 & $\mathrm{CH} 2$ & $1.096 \mathrm{~F}$ & DI3 & 123.5613 \\
\hline DI3 & 123.2975 & $\mathrm{CH} 3$ & 1.5124 & DI4 & 22.701 \\
\hline DI4 & 40.1519 & $\mathrm{CH} 4$ & 1.1126 & DI5 & 241.6301 \\
\hline DI5 & 117.5638 & CH5 & 1.0978 & DI6 & -126.0716 \\
\hline DI6 & -123.9825 & $\mathrm{CH} 6$ & 1.1043 & DI7 & 300.0324 \\
\hline DI7 & -35.67 & HPH & 120.037 & DI8 & 114.941 \\
\hline DI8 & -145.4247 & NPH & 103.6868 & DI9 & -209.3294 \\
\hline & & HNP1 & 110.6666 & DI10 & 60.1151 \\
\hline$B 3 L Y I$ & $/(D E L)$ & HNP2 & 111.1791 & DI11 & 120.555 \\
\hline $\mathrm{P}$ & & HNP3 & 110.7345 & DI12 & -121.4013 \\
\hline C 1 PF & & HNP4 & 107.2533 & & \\
\hline N 1 PI & I2 $2 \mathrm{HPH}$ & $\mathrm{OPH}$ & 117.7636 & $\mathrm{C}_{3} \mathrm{H}_{4} \mathrm{P}$ & $\mathrm{O}\left(\mathrm{NH}_{2}\right)_{2}{ }^{-}$ \\
\hline N 1 PI & 2 NPH 3 DI1 & HCP1 & 116.061 & & \\
\hline $\mathrm{H} 4 \mathrm{~N}$ & H1 1 HNP1 2 DI2 & HCP2 & $108.5752 \mathrm{~F}$ & $B 3 L Y P$ & \\
\hline $\mathrm{H} 4 \mathrm{~N}$ & H2 1 HNP2 5 DI3 & $\mathrm{HCP} 3$ & 121.7543 & $\mathrm{C}$ & \\
\hline H $3 \mathrm{~N}$ & H3 1 HNP3 2 DI4 & $\mathrm{HCC} 1$ & 115.7512 & $\mathrm{C} 1 \mathrm{CC}$ & \\
\hline H $3 \mathrm{~N}$ & H4 1 HNP4 7 DI5 & $\mathrm{HCC} 2$ & 110.121 & $\mathrm{C} 2 \mathrm{CC}$ & $\mathrm{C} 11 \mathrm{CCC}$ \\
\hline $\mathrm{O} 1 \mathrm{PC}$ & 2 OPH 3 DI6 & $\mathrm{HCC} 3$ & 112.0704 & $\mathrm{H} 1 \mathrm{CF}$ & H1 2 HCC1 3 DI 1 \\
\hline $\mathrm{H} 2 \mathrm{Cl}$ & I1 1 HCP1 3 DI7 & DI1 & 102.4332 & $\mathrm{H} 1 \mathrm{CF}$ & H2 2 HCC2 4 DI2 \\
\hline $\mathrm{H} 2 \mathrm{Cl}$ & I2 1 HCP2 10 DI8 & DI2 & 64.5119 & $\mathrm{H} 2 \mathrm{CH}$ & H3 1 HCC3 4 DI3 \\
\hline & & $\mathrm{DI} 3$ & 123.6658 & $\mathrm{H} 3 \mathrm{CH}$ & $\mathrm{H} 42 \mathrm{HCC} 46$ DI4 \\
\hline
\end{tabular}




\begin{tabular}{|c|c|}
\hline \multirow{8}{*}{\multicolumn{2}{|c|}{$\begin{array}{l}\text { P } 3 \text { CH5 } 2 \text { HCC5 } 7 \text { DI5 } \\
\text { N } 8 \text { PN } 3 \text { NPC } 2 \text { DI6 } \\
\text { N } 8 \text { PH } 3 \text { HPC } 9 \text { DI7 } \\
\text { H } 9 \text { NH1 } 8 \text { HNP } 10 \text { DI8 } \\
\text { H } 9 \text { NH2 } 8 \text { HNP2 } 10 \text { DI9 } \\
\text { H } 10 \text { NH3 } 8 \text { HNP3 } 9 \text { DI10 } \\
\text { H } 10 \text { NH4 } 8 \text { HNP4 } 13 \text { DI11 } \\
\text { O } 8 \text { PO } 3 \text { OPC } 9 \text { DI12 }\end{array}$}} \\
\hline & \\
\hline & \\
\hline & \\
\hline & \\
\hline & \\
\hline & \\
\hline & \\
\hline $\mathrm{CC}$ & 1.3748 \\
\hline $\mathrm{CC} 1$ & 1.422 \\
\hline CH1 & 1.0891 \\
\hline $\mathrm{CH} 2$ & 1.0869 \\
\hline $\mathrm{CH} 3$ & 1.0947 \\
\hline $\mathrm{CH} 4$ & 1.0887 \\
\hline $\mathrm{CH} 5$ & 1.7283 \\
\hline $\mathrm{PN}$ & 1.7262 \\
\hline $\mathrm{PH}$ & 1.7592 \\
\hline NH1 & 1.0147 \\
\hline NH2 & 1.0159 \\
\hline NH3 & 1.017 \\
\hline NH4 & 1.0178 \\
\hline PO & 1.5167 \\
\hline $\mathrm{CCC}$ & 129.6526 \\
\hline $\mathrm{HCC} 1$ & 121.9113 \\
\hline $\mathrm{HCC} 2$ & 121.0009 \\
\hline $\mathrm{HCC} 3$ & 115.4095 \\
\hline $\mathrm{HCC} 4$ & 119.8845 \\
\hline $\mathrm{HCC} 5$ & 125.4331 \\
\hline NPC & 104.8594 \\
\hline HPC & 116.8919 \\
\hline HNP & 111.7688 \\
\hline HNP2 & 112.1212 \\
\hline HNP3 & 107.7931 \\
\hline HNP4 & 111.4615 \\
\hline OPC & 116.1455 \\
\hline DI1 & -0.6342 \\
\hline DI2 & 178.7193 \\
\hline DI3 & 180.4611 \\
\hline DI4 & 181.3606 \\
\hline DI5 & 170.6297 \\
\hline DI6 & 39.1454 \\
\hline DI7 & -103.0616 \\
\hline DI8 & -179.8885 \\
\hline DI9 & -54.5779 \\
\hline DI10 & 160.3756 \\
\hline DI11 & 118.7229 \\
\hline DI12 & 131.7623 \\
\hline$M P 2$ & \\
\hline $\mathrm{C}$ & \\
\hline $\mathrm{C} 1 \mathrm{CC}$ & \\
\hline $\mathrm{C} 2 \mathrm{CC}$ & $11 \mathrm{CCC}$ \\
\hline $\mathrm{H} 1 \mathrm{CH}$ & I1 2 HCC1 3 DI1 \\
\hline $\mathrm{H} 1 \mathrm{CH}$ & I2 $2 \mathrm{HCC} 24 \mathrm{DI} 2$ \\
\hline $\mathrm{H} 2 \mathrm{CH}$ & $131 \mathrm{HCC} 34 \mathrm{DI} 3$ \\
\hline $\mathrm{H} 3 \mathrm{CF}$ & I4 2 HCC4 6 DI4 \\
\hline P $3 \mathrm{CH}$ & 52 HCC 57 DI5 \\
\hline N 8 PN & 3 NPC 2 DI6 \\
\hline N $8 \mathrm{PH}$ & [ 3 HPC 9 DI7 \\
\hline $\mathrm{H} 9 \mathrm{NH}$ & 118 HNP 10 DI8 \\
\hline $\mathrm{H} 9 \mathrm{NH}$ & I 8 HNP2 10 DI9 \\
\hline $\mathrm{H} 10 \mathrm{~N}$ & H3 8 HNP3 9 DI10 \\
\hline $\mathrm{H} 10 \mathrm{~N}$ & H4 8 HNP4 13 DI11 \\
\hline $\mathrm{O} 8 \mathrm{PC}$ & 3 OPC 9 DI12 \\
\hline $\mathrm{CC}$ & 1.3742 \\
\hline $\mathrm{CC} 1$ & 1.4233 \\
\hline $\mathrm{CH} 1$ & 1.0847 \\
\hline $\mathrm{CH} 2$ & 1.0827 \\
\hline $\mathrm{CH} 3$ & 1.0914 \\
\hline $\mathrm{CH} 4$ & 1.0856 \\
\hline CH5 & 1.7206 \\
\hline PN & 1.718 \\
\hline $\mathrm{PH}$ & 1.75 \\
\hline NH1 & 1.0132 \\
\hline $\mathrm{NH} 2$ & 1.0143 \\
\hline
\end{tabular}

$\begin{array}{lc}\text { NH3 } & 1.0155 \\ \text { NH4 } & 1.0165 \\ \text { PO } & 1.5216 \\ \text { CCC } & 129.1655 \\ \text { HCC1 } & 121.6882 \\ \text { HCC2 } & 120.8608 \\ \text { HCC3 } & 115.6193 \\ \text { HCC4 } & 119.6259 \\ \text { HCC5 } & 123.7248 \\ \text { NPC } & 103.6928 \\ \text { HPC } & 117.4552 \\ \text { HNP } & 111.6029 \\ \text { HNP2 } & 112.0626 \\ \text { HNP3 } & 108.0898 \\ \text { HNP4 } & 111.3511 \\ \text { OPC } & 116.4349 \\ \text { DI1 } & -0.8406 \\ \text { DI2 } & 177.7464 \\ \text { DI3 } & 180.9484 \\ \text { DI4 } & 183.4527 \\ \text { DI5 } & 163.8783 \\ \text { DI6 } & 42.2274 \\ \text { DI7 } & -101.9159 \\ \text { DI8 } & -180.7399 \\ \text { DI9 } & -55.6183 \\ \text { DI10 } & 163.955 \\ \text { DI11 } & 118.9893 \\ \text { DI12 } & 132.0294 \\ & \end{array}$

\section{$\mathrm{CH}_{3} \mathrm{PHOH}$}

B3LYP

C

P 1 PH1

O 2 PH $1 \mathrm{HPC}$

H 2 PH2 1 HPC2 3 di5

$\mathrm{H} 3 \mathrm{OH} 12 \mathrm{POH} 4$ di 6

H 1 CH1 2 HPC3 3 di7

H 1 CH2 2 HPC4 6 di8

H 1 CH3 2 HPC5 6 di9

$\begin{array}{lc}\text { PH1 } & 1.8537 \\ \text { PH } & 1.6944 \\ \text { PH2 } & 1.4279 \\ \text { OH1 } & 0.9652 \\ \text { CH1 } & 1.0939 \\ \text { CH2 } & 1.0935 \\ \text { CH3 } & 1.0963 \\ \text { HPC } & 99.1144 \\ \text { HPC2 } & 95.5096 \\ \text { POH } & 110.2052 \\ \text { HPC3 } & 111.5569 \\ \text { HPC4 } & 109.8374 \\ \text { HPC5 } & 108.806 \\ \text { di5 } & 98.6145 \\ \text { di6 } & 127.0527 \\ \text { di7 } & -43.2328 \\ \text { di8 } & 121.209 \\ \text { di9 } & -120.4136\end{array}$

$M P 2$

C

P $1 \mathrm{PH} 1$

O 2 PH 1 HPC

H 2 PH2 1 HPC2 3 di5

$\mathrm{H} 3 \mathrm{OH} 12 \mathrm{POH} 4$ di6

H 1 CH1 2 HPC3 3 di 7

H 1 CH2 2 HPC4 6 di8

H 1 CH3 2 HPC5 6 di9

$\begin{array}{lc}\text { PH1 } & 1.8364 \\ \text { PH } & 1.6955 \\ \text { PH2 } & 1.4103 \\ \text { OH1 } & 0.9656 \\ \text { CH1 } & 1.0888 \\ \text { CH2 } & 1.0882 \\ \text { CH3 } & 1.0914\end{array}$

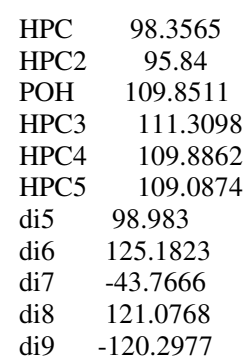

$\mathrm{CH}_{2} \mathrm{PHOH}^{-}$

B3LYP

$\mathrm{P}$

O 1 PO

$\mathrm{H} 1 \mathrm{PH} 2 \mathrm{HPO}$

H 2 OH 1 HOP 3 DI

$\mathrm{C} 1 \mathrm{PC} 2 \mathrm{CPO} 3 \mathrm{DI} 2$

H 5 CH1 1 HCP1 2 DI3

H 5 CH2 1 HCP2 6 DI4

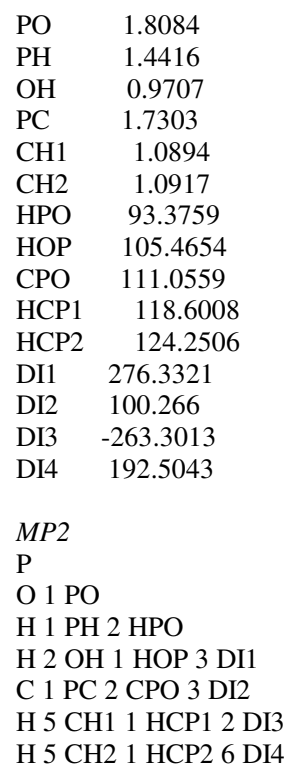

$\begin{array}{lc}\text { PO } & 1.8107 \\ \text { PH } & 1.4223 \\ \text { OH } & 0.9718 \\ \text { PC } & 1.7234 \\ \text { CH1 } & 1.0846 \\ \text { CH2 } & 1.087 \\ \text { HPO } & 93.2531 \\ \text { HOP } & 104.5809 \\ \text { CPO } & 111.0352 \\ \text { HCP1 } & 118.869 \\ \text { HCP2 } & 123.691 \\ \text { DI1 } & 277.756 \\ \text { DI2 } & 99.8067 \\ \text { DI3 } & -263.1366 \\ \text { DI4 } & 192.1658\end{array}$

$\mathrm{C}_{2} \mathrm{H}_{4} \mathrm{PHOH}^{-}$

B3LYP

C

P 1 PH1

O 2 PH 1 HPC

H 2 PH2 1 HPC2 3 di5

$\mathrm{H} 3 \mathrm{OH} 12 \mathrm{POH} 4$ di6

$\mathrm{X} 1 \mathrm{CH} 12 \mathrm{HPC} 33 \mathrm{di} 7$

H 1 CH2 2 HPC4 6 di8

C 1 CH3 2 HPC5 6 di9

H 8 CH4 1 HCC1 6 dilo

H 8 CH5 1 HCC2 9 dil1 
H 8 CH6 1 HCC3 9 di12

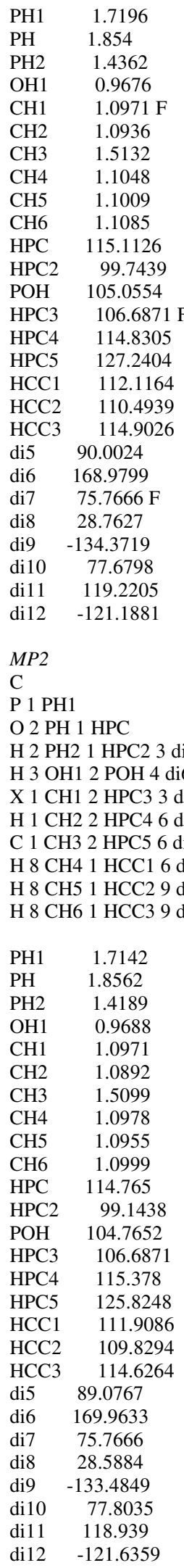

$\mathrm{C}_{3} \mathrm{H}_{4} \mathrm{PHOH}^{-}$

B3LYP

$\mathrm{C}$

$\mathrm{C} 1 \mathrm{CC}$

C 2 CC $11 \mathrm{CCC}$
H 1 CH1 2 HCC1 3 DI 1

$\mathrm{H} 1 \mathrm{CH} 22 \mathrm{HCC} 24 \mathrm{DI} 2$

$\mathrm{H} 2 \mathrm{CH} 31 \mathrm{HCC} 34$ DI3

$\mathrm{H} 3 \mathrm{CH} 42 \mathrm{HCC} 46$ DI 4

P 3 CH5 2 HCC5 7 DI5

O 8 PO 3 OPC 7 DI6

H 8 PH 3 HPC 9 DI7

H 9 OH 8 HOP 10 DI8

$\begin{array}{ll}\text { CC } & 1.3731 \\ \text { CC1 } & 1.425 \\ \text { CH1 } & 1.0892 \\ \text { CH2 } & 1.0872 \\ \text { CH3 } & 1.0968 \\ \text { CH4 } & 1.0928 \\ \text { CH5 } & 1.7457 \\ \text { PO } & 1.796 \\ \text { PH } & 1.4294 \\ \text { OH } & 0.9674 \\ \text { CCC } & 129.7348 \\ \text { HCC1 } & 121.7596 \\ \text { HCC2 } & 121.22 \\ \text { HCC3 } & 115.7372 \\ \text { HCC4 } & 117.6833 \\ \text { HCC5 } & 120.9902 \\ \text { OPC } & 111.0787 \\ \text { HPC } & 100.5529 \\ \text { HOP } & 106.3807 \\ \text { DI1 } & 1.5463 \\ \text { DI2 } & 180.7431 \\ \text { DI3 } & 180.5227 \\ \text { DI4 } & 179.8081 \\ \text { DI5 } & 185.5521 \\ \text { DI6 } & -64.1829 \\ \text { DI7 } & 91.6913 \\ \text { DI8 } & 175.7422 \\ & \end{array}$

$M P 2$

$\mathrm{C}$

C 1 CC

C 2 CC $11 \mathrm{CCC}$

H 1 CH1 2 HCC1 3 DI 1

$\mathrm{H} 1 \mathrm{CH} 22 \mathrm{HCC} 24 \mathrm{DI} 2$

$\mathrm{H} 2 \mathrm{CH} 31 \mathrm{HCC} 34 \mathrm{DI} 3$

H 3 CH4 2 HCC4 6 DI4

P 3 CH5 2 HCC5 7 DI5

O 8 PO 3 OPC 7 DI6

H 8 PH 3 HPC 9 DI7

H 9 OH 8 HOP 10 DI8

$\begin{array}{ll}\text { CC } & 1.371 \\ \text { CC1 } & 1.4281 \\ \text { CH1 } & 1.0847 \\ \text { CH2 } & 1.0829 \\ \text { CH3 } & 1.0933 \\ \text { CH4 } & 1.0892 \\ \text { CH5 } & 1.7344 \\ \text { PO } & 1.797 \\ \text { PH } & 1.4129 \\ \text { OH } & 0.9682 \\ \text { CCC } & 129.2261 \\ \text { HCC1 } & 121.5579 \\ \text { HCC2 } & 121.1055 \\ \text { HCC3 } & 116.0465 \\ \text { HCC4 } & 117.8669 \\ \text { HCC5 } & 119.9741 \\ \text { OPC } & 111.475 \\ \text { HPC } & 100.5723 \\ \text { HOP } & 105.9144 \\ \text { DI1 } & 1.4255 \\ \text { DI2 } & 181.962 \\ \text { DI3 } & 180.0856 \\ \text { DI4 } & 180.5405 \\ \text { DI5 } & 187.7246 \\ \text { DI6 } & -63.9661 \\ \text { DI7 } & 91.6468 \\ \text { DI8 } & 175.0331 \\ & \end{array}$

$\mathrm{CH}_{3} \mathrm{P}(\mathrm{OH})_{2}$

$B 3 L Y P$

$\mathrm{C}$

P 1 PH1

O 2 PH 1 HPC

O 2 PH2 1 HPC2 3 di5

H 3 OH1 2 POH 4 di6

$\mathrm{H} 4 \mathrm{OH} 22 \mathrm{POH} 23$ di 7

H 1 CH1 2 HCP1 3 di8

$\mathrm{H} 1 \mathrm{CH} 22 \mathrm{HCP} 27$ di9

H 1 CH3 2 HCP3 7 di10

PH1 1.8346

PH 1.6805

PH2 1.6805

$\mathrm{OH} 1 \quad 0.9662$

$\mathrm{OH} 2 \quad 0.9662$

CH1 1.0949

CH2 1.0935

$\mathrm{CH} 3 \quad 1.0935$

HPC 95.3391

HPC2 95.358

POH 111.2843

POH2 111.2755

HCP1 109.4925

HCP2 109.5593

HCP3 109.5595

di5 103.8014

di6 103.8977

di7 -104.1027

di8 $\quad-51.9088$

di9 120.0737

di10 -120.0797

$M P 2$

$\mathrm{C}$

P 1 PH1

O 2 PH 1 HPC

O 2 PH2 1 HPC2 3 di5

H 3 OH1 2 POH 4 di 6

$\mathrm{H} 4 \mathrm{OH} 22 \mathrm{POH} 23$ di 7

H 1 CH1 2 HCP1 3 di8

$\mathrm{H} 1 \mathrm{CH} 22 \mathrm{HCP} 27$ di9

H 1 CH3 2 HCP3 7 di10

$\begin{array}{lc}\text { PH1 } & 1.8177 \\ \text { PH } & 1.6797 \\ \text { PH2 } & 1.6797 \\ \text { OH1 } & 0.9668 \\ \text { OH2 } & 0.9668 \\ \text { CH1 } & 1.09 \\ \text { CH2 } & 1.0886 \\ \text { CH3 } & 1.0886 \\ \text { HPC } & 95.0154 \\ \text { HPC2 } & 95.0254 \\ \text { POH } & 110.868 \\ \text { POH2 } & 110.8616 \\ \text { HCP1 } & 109.0599 \\ \text { HCP2 } & 109.6909 \\ \text { HCP3 } & 109.6882 \\ \text { di5 } & 103.9619 \\ \text { di6 } & 104.7934 \\ \text { di7 } & -104.9063 \\ \text { di8 } & -52.0025 \\ \text { di9 } & 119.9896 \\ \text { di10 } & -119.9844\end{array}$

$\mathrm{CH}_{2} \mathrm{P}(\mathrm{OH})_{2}^{-}$

B3LYP

$\mathrm{P}$

O 1 PO1

$\mathrm{O} 1 \mathrm{PO} 22 \mathrm{OPO}$

H 2 OH1 1 HOP1 3 DI 1

$\mathrm{H} 3 \mathrm{OH} 21 \mathrm{HOP} 22 \mathrm{DI} 2$ 
O 8 PO2 3 OPC2 9 DI7

H 9 OH1 8 HOP1 10 DI8

H 10 OH2 8 HOP2 9 DI9

$\begin{array}{lc}\text { CC } & 1.3699 \\ \text { CC1 } & 1.4281 \\ \text { CH1 } & 1.0846 \\ \text { CH2 } & 1.0826 \\ \text { CH3 } & 1.0924 \\ \text { CH4 } & 1.0922 \\ \text { CH5 } & 1.7306 \\ \text { PO1 } & 1.7602 \\ \text { PO2 } & 1.7252 \\ \text { OH1 } & 0.9692 \\ \text { OH2 } & 0.9717 \\ \text { CCC } & 129.1583 \\ \text { HCC1 } & 121.6189 \\ \text { HCC2 } & 121.0193 \\ \text { HCC3 } & 115.9722 \\ \text { HCC4 } & 118.498 \\ \text { HCC5 } & 120.1333 \\ \text { OPC1 } & 100.4404 \\ \text { OPC2 } & 109.8635 \\ \text { HOP1 } & 103.3384 \\ \text { HOP2 } & 107.6321 \\ \text { DI1 } & -1.0102 \\ \text { DI2 } & 179.8801 \\ \text { DI3 } & 179.9994 \\ \text { DI4 } & 183.8943 \\ \text { DI5 } & 169.4112 \\ \text { DI6 } & 310.2697 \\ \text { DI7 } & 93.7213 \\ \text { DI8 } & 217.353 \\ \text { DI9 } & 35.533 \\ & \end{array}$

$\mathrm{CH}_{3} \mathrm{PO}(\mathrm{OH})_{2}$

$B 3 L Y P$
P
O 1 PO
O 1 PO1 2 OPO1
O 11 PO2 22 OPO2 3 DI1
H 3 OH1 1 POH1 4 DI2
H 4 OH2 1 POH2 3 DI3
C 1 PC 2 CPO 3 DI4
H 7 CH1 1 HCP1 2 DI5
H 7 CH2 1 HCP2 2 DI6
H 7 CH3 11 HCP3 2 DI7

PO $\quad 1.4898$

PO1 1.6307

$\mathrm{PO} 21.6303$

OH1 0.9689

$\mathrm{OH} 2 \quad 0.9689$

PC 1.8021

CH1 1.0921

$\mathrm{CH} 2 \quad 1.0921$

CH3 1.0927

OPO1 113.5117

$\mathrm{OPO} 2113.6446$

POH1 111.1307

POH2 111.231

CPO 119.2762

HCP1 109.0483

HCP2 109.0296

HCP3 110.0014

DI1 $\quad-119.9621$

DI2 108.0796

DI3 $\quad-106.4551$

DI4 120.0569

DI5 59.9415

DI6 -59.7522

DI7 180.1056

$M P 2$

$\mathrm{P}$

O 1 PO
O 1 PO1 2 OPO1

O 1 PO2 2 OPO2 3 DI

$\mathrm{H} 3 \mathrm{OH} 11 \mathrm{POH} 14 \mathrm{DI} 2$

$\mathrm{H} 4 \mathrm{OH} 21 \mathrm{POH} 23 \mathrm{DI} 3$

C 1 PC 2 CPO 3 DI4

H 7 CH1 1 HCP1 2 DI5

H 7 CH2 1 HCP2 2 DI6

$\mathrm{H} 7 \mathrm{CH} 31$ HCP3 2 DI7

$\begin{array}{lc}\text { PO } & 1.4945 \\ \text { PO1 } & 1.6314 \\ \text { PO2 } & 1.631 \\ \text { OH1 } & 0.9693 \\ \text { OH2 } & 0.9693 \\ \text { PC } & 1.7895 \\ \text { CH1 } & 1.0873 \\ \text { CH2 } & 1.0873 \\ \text { CH3 } & 1.0882 \\ \text { OPO1 } & 113.811 \\ \text { OPO2 } & 113.9246 \\ \text { POH1 } & 110.7568 \\ \text { POH2 } & 110.8531 \\ \text { CPO } & 119.5597 \\ \text { HCP1 } & 109.1363 \\ \text { HCP2 } & 109.1205 \\ \text { HCP3 } & 109.6431 \\ \text { DI1 } & -120.4722 \\ \text { DI2 } & 107.4203 \\ \text { DI3 } & -105.9823 \\ \text { DI4 } & 119.8125 \\ \text { DI5 } & 59.982 \\ \text { DI6 } & -59.9577 \\ \text { DI7 } & 180.0205\end{array}$

$\mathrm{CH}_{2} \mathrm{PO}(\mathrm{OH})_{2}$

B3LYP

$\mathrm{P}$

$\mathrm{O} 1 \mathrm{PO}$

O 1 PO1 2 OPO 1

O 1 PO2 2 OPO2 3 DI

$\mathrm{H} 3 \mathrm{OH} 11 \mathrm{POH} 14 \mathrm{DI} 2$

$\mathrm{H} 4 \mathrm{OH} 21 \mathrm{POH} 23$ DI3

C 1 PC 2 CPO 3 DI 4

H 7 CH1 1 HCP1 2 DI5

H 7 CH2 1 HCP2 8 DI6

$\begin{array}{lc}\text { PO } & 1.5139 \\ \text { PO1 } & 1.6814 \\ \text { PO2 } & 1.6929 \\ \text { OH1 } & 0.9683 \\ \text { OH2 } & 0.9685 \\ \text { PC } & 1.7016 \\ \text { CH1 } & 1.0897 \\ \text { CH2 } & 1.0878 \\ \text { OPO1 } & 112.6852 \\ \text { OPO2 } & 105.8018 \\ \text { POH1 } & 103.82 \\ \text { POH2 } & 105.6699 \\ \text { CPO } & 120.0578 \\ \text { HCP1 } & 116.8956 \\ \text { HCP2 } & 115.6979 \\ \text { DI1 } & -102.8185 \\ \text { DI2 } & 204.5139 \\ \text { DI3 } & -95.1941 \\ \text { DI4 } & 123.7687 \\ \text { DI5 } & 176.2466 \\ \text { DI6 } & 218.618\end{array}$

$M P 2$

$\mathrm{P}$

O 1 PO1

$\mathrm{O} 1 \mathrm{PO} 22 \mathrm{OPO} 1$

O 1 PO3 2 OPO2 3 DI

H 3 OH1 1 HOP1 2 DI 2

$\mathrm{H} 4 \mathrm{OH} 21 \mathrm{HOP} 22 \mathrm{DI} 3$

$\mathrm{C} 1 \mathrm{PC} 2 \mathrm{OPC} 3 \mathrm{DI} 4$

$\mathrm{H} 7 \mathrm{CH} 11 \mathrm{HCP} 12 \mathrm{DI} 5$

H 7 CH2 1 HCP2 8 DI6

$\begin{array}{lc}\text { PO1 } & 1.5182 \\ \text { PO2 } & 1.6805 \\ \text { PO3 } & 1.6922 \\ \text { OH1 } & 0.9687 \\ \text { OH2 } & 0.9688 \\ \text { PC } & 1.6963 \\ \text { CH1 } & 1.0837 \\ \text { CH2 } & 1.0857 \\ \text { OPO1 } & 113.1494 \\ \text { OPO2 } & 105.8648 \\ \text { HOP1 } & 103.4564 \\ \text { HOP2 } & 105.2573 \\ \text { OPC } & 120.0847 \\ \text { HCP1 } & 115.4011 \\ \text { HCP2 } & 115.8366 \\ \text { DI1 } & -102.8489 \\ \text { DI2 } & 94.4535 \\ \text { DI3 } & 20.7312 \\ \text { DI4 } & 123.5119 \\ \text { DI5 } & 37.5251 \\ \text { DI6 } & 139.5436\end{array}$

$\mathrm{C}_{2} \mathrm{H}_{4} \mathrm{PO}(\mathrm{OH})_{2}{ }^{-}$

B3LYP

$\mathrm{P}$

O 1 PO

O 1 PO1 2 OPO1

O 1 PO2 2 OPO2 3 DI

H 3 OH1 1 HOP1 4 DI2

$\mathrm{H} 4 \mathrm{OH} 21 \mathrm{HOP} 23 \mathrm{DI} 3$

C 1 PC 2 CPO 3 DI 4

C 7 CC 1 CCP 2 DI5

X 7 CH1 1 HCP1 8 DI6

$\mathrm{H} 7 \mathrm{CH} 21 \mathrm{HCP} 28 \mathrm{DI} 7$

H 8 CH3 7 HCC1 10 DI8

H 8 CH4 7 HCC2 10 DI9

H 8 CH5 7 HCC3 10 DI10

$\begin{array}{lc}\text { PO } & 1.5159 \\ \text { PO1 } & 1.6974 \\ \text { PO2 } & 1.6781 \\ \text { OH1 } & 0.9684 \\ \text { OH2 } & 0.9686 \\ \text { PC } & 1.7001 \\ \text { CC } & 1.5155 \\ \text { CH1 } & 1.0954 \mathrm{~F} \\ \text { CH2 } & 1.092 \\ \text { CH3 } & 1.0972 \\ \text { CH4 } & 1.1101 \\ \text { CH5 } & 1.1027 \\ \text { OPO1 } & 105.1261 \\ \text { OPO2 } & 113.6265 \\ \text { HOP1 } & 105.6155 \\ \text { HOP2 } & 103.7238 \\ \text { CPO } & 119.7054 \\ \text { CCP } & 120.9955 \\ \text { HCP1 } & 105.8927 \mathrm{~F} \\ \text { HCP2 } & 114.203 \\ \text { HCC1 } & 110.3926 \\ \text { HCC2 } & 115.2814 \\ \text { HCC3 } & 111.5857 \\ \text { DI1 } & 102.5891 \\ \text { DI2 } & -98.2689 \\ \text { DI3 } & 202.678 \\ \text { DI4 } & -133.2443 \\ \text { DI5 } & 35.117 \\ \text { DI6 } & -122.3073 \mathrm{~F} \\ \text { DI7 } & 147.5687 \\ \text { DI8 } & -179.1998 \\ \text { DI9 } & -58.4803 \\ \text { DI10 } & 62.5694 \\ & \\ \text { MP2 } & \\ & \end{array}$


$\mathrm{P}$

$\mathrm{O} 1 \mathrm{PO}$

O 1 PO1 2 OPO1

O 1 PO2 2 OPO2 3 DI 1

H 3 OH1 1 HOP1 4 DI2

$\mathrm{H} 4 \mathrm{OH} 21 \mathrm{HOP} 23 \mathrm{DI} 3$

$\mathrm{C} 1 \mathrm{PC} 2 \mathrm{CPO} 3 \mathrm{DI} 4$

C 7 CC 1 CCP 2 DI5

$\mathrm{X} 7 \mathrm{CH} 11$ HCP1 8 DI6

$\mathrm{H} 7 \mathrm{CH} 21 \mathrm{HCP} 28$ DI7

H 8 CH3 7 HCC1 10 DI8

H 8 CH4 7 HCC2 10 DI9

H 8 CH5 7 HCC3 10 DI10

$\begin{array}{lc}\text { PO } & 1.5204 \\ \text { PO1 } & 1.6974 \\ \text { PO2 } & 1.6769 \\ \text { OH1 } & 0.9689 \\ \text { OH2 } & 0.9691 \\ \text { PC } & 1.6945 \\ \text { CC } & 1.5131 \\ \text { CH1 } & 1.0954 \\ \text { CH2 } & 1.0881 \\ \text { CH3 } & 1.0925 \\ \text { CH4 } & 1.1019 \\ \text { CH5 } & 1.096 \\ \text { OPO1 } & 105.0994 \\ \text { OPO2 } & 114.0922 \\ \text { HOP1 } & 105.1771 \\ \text { HOP2 } & 103.2038 \\ \text { CPO } & 119.5285 \\ \text { CCP } & 119.7478 \\ \text { HCP1 } & 105.8927 \\ \text { HCP2 } & 113.7301 \\ \text { HCC1 } & 109.9084 \\ \text { HCC2 } & 114.9496 \\ \text { HCC3 } & 111.3646 \\ \text { DI1 } & 102.7643 \\ \text { DI2 } & -98.0516 \\ \text { DI3 } & 202.4993 \\ \text { DI4 } & -133.142 \\ \text { DI5 } & 37.6384 \\ \text { DI6 } & -122.3073 \\ \text { DI7 } & 144.7056 \\ \text { DI8 } & -177.2139 \\ \text { DI9 } & -56.5789 \\ \text { DI10 } & 64.665\end{array}$

$\mathrm{C}_{3} \mathrm{H}_{4} \mathrm{PO}(\mathrm{OH})_{2}{ }^{-}$

\begin{tabular}{|c|c|}
\hline $3 L$ & \\
\hline $\mathrm{C} 1 \mathrm{C}$ & \\
\hline C $2 C$ & $1 \mathrm{CCC}$ \\
\hline & $2 \mathrm{HCC}$ \\
\hline & To \\
\hline & $1 \mathrm{HCC}$ \\
\hline C & $2 \mathrm{HCC}$ \\
\hline $\mathrm{C}$ & $52 \mathrm{HCC}$ \\
\hline $8 \mathrm{P}$ & $3 \mathrm{OPC}$ \\
\hline 8 & OPC \\
\hline $8 \mathrm{P}$ & 3 OPC \\
\hline 10 & $18 \mathrm{HO}$ \\
\hline & \\
\hline $\mathrm{C}$ & 1.3691 \\
\hline $\mathrm{C} 1$ & 1.426 \\
\hline CH1 & 1.0893 \\
\hline $\mathrm{CH} 2$ & 1.0866 \\
\hline H3 & 1.0928 \\
\hline CH4 & 1.0895 \\
\hline CH5 & 1.7136 \\
\hline $\mathrm{O} 1$ & 1.5093 \\
\hline $\mathrm{O} 2$ & 1.6759 \\
\hline 3 & 1.6725 \\
\hline $\mathrm{H} 1$ & \\
\hline & \\
\hline
\end{tabular}

$\begin{array}{lc}\text { CCC } & 128.961 \\ \text { HCC1 } & 121.9612 \\ \text { HCC2 } & 120.9973 \\ \text { HCC3 } & 116.355 \\ \text { HCC4 } & 119.6242 \\ \text { HCC5 } & 122.7448 \\ \text { OPC1 } & 119.478 \\ \text { OPC2 } & 113.3983 \\ \text { OPC3 } & 105.1992 \\ \text { HOP } & 105.9689 \\ \text { HOP2 } & 105.951 \\ \text { DI1 } & 1.3522 \\ \text { DI2 } & 180.7503 \\ \text { DI3 } & 179.7919 \\ \text { DI4 } & 176.2973 \\ \text { DI5 } & 192.4316 \\ \text { DI6 } & 172.0847 \\ \text { DI7 } & 127.7828 \\ \text { DI8 } & -129.5181 \\ \text { DI9 } & 104.6533 \\ \text { DI10 } & -200.4225 \\ & \end{array}$

$M P 2$

$\mathrm{C}$

$\mathrm{C} 1 \mathrm{CC}$

C 2 CC $11 \mathrm{CCC}$

H 1 CH1 2 HCC1 3 DI

$\mathrm{H} 1 \mathrm{CH} 22 \mathrm{HCC} 24 \mathrm{DI} 2$

$\mathrm{H} 2 \mathrm{CH} 31 \mathrm{HCC} 34 \mathrm{DI} 3$

H 3 CH4 2 HCC4 6 DI4

P 3 CH5 2 HCC5 7 DI5

O 8 PO1 3 OPC1 7 DI6

O 8 PO2 3 OPC2 9 DI7

O 8 PO3 3 OPC 39 DI8

H 10 OH1 8 HOP 11 DI9

H 11 OH2 8 HOP2 10 DI10

$\begin{array}{lc}\text { CC } & 1.3686 \\ \text { CC1 } & 1.4282 \\ \text { CH1 } & 1.0848 \\ \text { CH2 } & 1.0823 \\ \text { CH3 } & 1.0894 \\ \text { CH4 } & 1.0868 \\ \text { CH5 } & 1.7117 \\ \text { PO1 } & 1.5124 \\ \text { PO2 } & 1.6794 \\ \text { PO3 } & 1.6643 \\ \text { OH1 } & 0.9677 \\ \text { OH2 } & 0.9682 \\ \text { CCC } & 128.457 \\ \text { HCC1 } & 121.768 \\ \text { HCC2 } & 120.8461 \\ \text { HCC3 } & 116.6933 \\ \text { HCC4 } & 119.2778 \\ \text { HCC5 } & 121.3918 \\ \text { OPC1 } & 117.4687 \\ \text { OPC2 } & 114.4923 \\ \text { OPC3 } & 105.5885 \\ \text { HOP } & 105.4479 \\ \text { HOP2 } & 104.5876 \\ \text { DI1 } & 1.5258 \\ \text { DI2 } & 180.8716 \\ \text { DI3 } & 179.6367 \\ \text { DI4 } & 174.1969 \\ \text { DI5 } & 198.8788 \\ \text { DI6 } & 182.0584 \\ \text { DI7 } & 129.0553 \\ \text { DI8 } & -130.9189 \\ \text { DI9 } & 153.3047 \\ \text { DI10 } & -208.4511 \\ & \end{array}$

$\mathrm{CH}_{3} \mathrm{PHF}$

$B 3 L Y P$

$\mathrm{C}$

$\mathrm{H} 1 \mathrm{CH} 1$

$\mathrm{H} 1 \mathrm{CH} 22 \mathrm{HCH}$
$\mathrm{H} 1 \mathrm{CH} 32 \mathrm{HCH} 23 \mathrm{DI}$ P 1 PC 2 PCH 3 DI2 F 5 PF 1 FPC 2 DI4 H 5 PH 1 HPC 6 DI5

$\begin{array}{lc}\text { CH1 } & 1.0934 \\ \text { CH2 } & 1.0933 \\ \text { CH3 } & 1.097 \\ \text { PC } & 1.8528 \\ \text { PF } & 1.6561 \\ \text { PH } & 1.4297 \\ \text { HCH } & 109.4555 \\ \text { HCH2 } & 107.9919 \\ \text { PCH } & 109.781 \\ \text { FPC } & 99.0889 \\ \text { HPC } & 95.6172 \\ \text { DI1 } & 118.6126 \\ \text { DI2 } & -123.6796 \\ \text { DI4 } & 75.7692 \\ \text { DI5 } & 97.3491\end{array}$

$M P 2$

$\mathrm{C}$ $\mathrm{H} 1 \mathrm{CH} 1$

$\mathrm{H} 1 \mathrm{CH} 22 \mathrm{HCH}$ H 1 CH3 2 HCH2 3 DI1 P 1 PC 2 PCH 3 DI 2 F 5 PF 1 FPC 2 DI4 H 5 PH 1 HPC 6 DI5

$\begin{array}{lc}\text { CH1 } & 1.088 \\ \text { CH2 } & 1.0882 \\ \text { CH3 } & 1.092 \\ \text { PC } & 1.8359 \\ \text { PF } & 1.6571 \\ \text { PH } & 1.411 \\ \text { HCH } & 109.486 \\ \text { HCH2 } & 108.0463 \\ \text { PCH } & 109.9806 \\ \text { FPC } & 98.8053 \\ \text { HPC } & 95.9573 \\ \text { DI1 } & 118.48 \\ \text { DI2 } & -123.5694 \\ \text { DI4 } & 76.3382 \\ \text { DI5 } & 97.4885\end{array}$

$\mathrm{CH}_{2} \mathrm{PHF}^{-}$

B3LYP

$\mathrm{C}$

$\mathrm{H} 1 \mathrm{CH} 1$

$\mathrm{H} 1 \mathrm{CH} 22 \mathrm{HCH}$

$\mathrm{X} 1 \mathrm{CH} 32 \mathrm{HCH} 23 \mathrm{DI} 1$

P 1 PC 2 PCH 3 DI2

F 5 PF 1 FPC 2 DI4

H 5 PH 1 HPC 6 DI5

\begin{tabular}{|c|c|}
\hline $\mathrm{CH} 1$ & 1.0908 \\
\hline $\mathrm{CH} 2$ & 1.0919 \\
\hline $\mathrm{CH} 3$ & 1.0964 \\
\hline PC & 1.7114 \\
\hline PF & 1.8327 \\
\hline PH & 1.4354 \\
\hline $\mathrm{HCH}$ & 115.1158 \\
\hline $\mathrm{HCH} 2$ & 82.8855 \\
\hline $\mathrm{PCH}$ & 117.5286 \\
\hline FPC & 113.7588 \\
\hline HPC & 99.0231 \\
\hline DI1 & 157.6374 \\
\hline DI2 & -161.3466 \\
\hline DI4 & 96.6094 \\
\hline DI5 & 91.575 \\
\hline \multicolumn{2}{|l|}{$M P 2$} \\
\hline \multicolumn{2}{|l|}{$\mathrm{C}$} \\
\hline \multicolumn{2}{|c|}{$\mathrm{H} 1 \mathrm{CH} 1$} \\
\hline
\end{tabular}




\begin{tabular}{|c|c|}
\hline $\mathrm{CH} 2$ & 1.0869 \\
\hline $\mathrm{CH} 3$ & $1.0964 \mathrm{~F}$ \\
\hline PC & 1.7054 \\
\hline PF & 1.8298 \\
\hline PH & 1.4169 \\
\hline $\mathrm{HCH}$ & 115.6998 \\
\hline $\mathrm{HCH} 2$ & $82.8855 \mathrm{~F}$ \\
\hline $\mathrm{PCH}$ & 117.7856 \\
\hline FPC & 113.5929 \\
\hline HPC & 98.8714 \\
\hline DI1 & $157.6374 \mathrm{~F}$ \\
\hline DI2 & -162.1928 \\
\hline DI4 & 97.5427 \\
\hline DI5 & 91.1436 \\
\hline
\end{tabular}

$\mathrm{C}_{2} \mathrm{H}_{4} \mathrm{PHF}^{-}$

B3LYP

$\mathrm{C}$

C $1 \mathrm{CC} 1$

$\mathrm{H} 1 \mathrm{CH} 12 \mathrm{HCC} 1$

H 1 CH2 2 HCC2 3 DI 1

$\mathrm{H} 1 \mathrm{CH} 32 \mathrm{HCC} 33 \mathrm{DI} 2$

$\mathrm{H} 2 \mathrm{CH} 41 \mathrm{HCC} 43 \mathrm{DI} 3$

P 2 CP 1 PCC 6 DI4

H 7 PH 2 HPC 6 DI5

F 7 PF 2 FPC 8 DI6

$\begin{array}{lc}\text { CC1 } & 1.5136 \\ \text { CH1 } & 1.1044 \\ \text { CH2 } & 1.0996 \\ \text { CH3 } & 1.1072 \\ \text { CH4 } & 1.0942 \\ \text { CP } & 1.7077 \\ \text { PH } & 1.4388 \\ \text { PF } & 1.8543 \\ \text { HCC1 } & 112.0624 \\ \text { HCC2 } & 110.5641 \\ \text { HCC3 } & 114.3405 \\ \text { HCC4 } & 115.2421 \\ \text { PCC } & 127.9203 \\ \text { HPC } & 98.4584 \\ \text { FPC } & 114.2635 \\ \text { DI1 } & 119.3787 \\ \text { DI2 } & -120.8202 \\ \text { DI3 } & 51.9979 \\ \text { DI4 } & 161.93 \\ \text { DI5 } & 190.2589 \\ \text { DI6 } & -90.3313\end{array}$

$M P 2$

$\mathrm{C}$

$\mathrm{C} 1 \mathrm{CC} 1$

$\mathrm{H} 1 \mathrm{CH} 12 \mathrm{HCC} 1$

$\mathrm{H} 1 \mathrm{CH} 22$ HCC2 3 DI 1

$\mathrm{H} 1 \mathrm{CH} 32 \mathrm{HCC} 33$ DI2

$\mathrm{H} 2 \mathrm{CH} 41$ HCC4 3 DI3

P 2 CP 1 PCC 6 DI4

H 7 PH 2 HPC 6 DI5

F 7 PF 2 FPC 8 DI6

$\begin{array}{ll}\mathrm{CC} 1 & 1.5099 \\ \mathrm{CH} 1 & 1.0975 \\ \mathrm{CH} 2 & 1.0944 \\ \mathrm{CH} 3 & 1.099 \\ \mathrm{CH} 4 & 1.0895 \\ \mathrm{CP} & 1.7038 \\ \mathrm{PH} & 1.4206 \\ \mathrm{PF} & 1.8535 \\ \mathrm{HCC} 1 & 111.8833 \\ \mathrm{HCC} 2 & 109.9265\end{array}$

$\begin{array}{lc}\text { HCC3 } & 114.056 \\ \text { HCC4 } & 115.796 \\ \text { PCC } & 126.6538 \\ \text { HPC } & 97.9241 \\ \text { FPC } & 113.7772 \\ \text { DI1 } & 119.1164 \\ \text { DI2 } & -121.272 \\ \text { DI3 } & 52.0224 \\ \text { DI4 } & 161.3262 \\ \text { DI5 } & 189.7159 \\ \text { DI6 } & -89.5297\end{array}$

$\mathrm{C}_{3} \mathrm{H}_{4} \mathrm{PHF}$

B3LYP

$\mathrm{C}$

$\mathrm{C} 1 \mathrm{CC}$

C 2 CC $11 \mathrm{CCC}$

H 1 CH1 2 HCC1 3 DI 1

$\mathrm{H} 1 \mathrm{CH} 22 \mathrm{HCC} 24$ DI2

$\mathrm{H} 2 \mathrm{CH} 31 \mathrm{HCC} 34 \mathrm{DI} 3$

$\mathrm{H} 3 \mathrm{CH} 42$ HCC4 6 DI 4

P 3 CH5 2 HCC5 7 DI5

F 8 PF 3 FPC 2 DI6

H 8 PH 3 HPC 9 DI7

$\begin{array}{lc}\mathrm{CC} & 1.3684 \\ \mathrm{CC} 1 & 1.4303 \\ \mathrm{CH} 1 & 1.0891 \\ \mathrm{CH} 2 & 1.0871 \\ \mathrm{CH} 3 & 1.096 \\ \mathrm{CH} 4 & 1.0921 \\ \mathrm{CH} 5 & 1.7319 \\ \mathrm{PF} & 1.7815 \\ \mathrm{PH} & 1.4328 \\ \mathrm{CCC} & 129.2033 \\ \mathrm{HCC} 1 & 121.6971 \\ \mathrm{HCC} 2 & 121.3017 \\ \mathrm{HCC} 3 & 116.0669 \\ \mathrm{HCC} 4 & 117.3022 \\ \mathrm{HCC} 5 & 121.0171 \\ \text { FPC } & 110.5537 \\ \mathrm{HPC} & 98.6856 \\ \mathrm{DI} 1 & 1.963 \\ \text { DI2 } & 180.259 \\ \text { DI3 } & 180.6752 \\ \text { DI4 } & 178.4358 \\ \text { DI5 } & 186.9907 \\ \text { DI6 } & 103.6238 \\ \text { DI7 } & 92.5323\end{array}$

\section{$M P 2$}

$\mathrm{C}$

$\mathrm{C} 1 \mathrm{CC}$

C 2 CC $11 \mathrm{CCC}$

H 1 CH1 2 HCC1 3 DI 1

$\mathrm{H} 1 \mathrm{CH} 22 \mathrm{HCC} 24$ DI2

$\mathrm{H} 2 \mathrm{CH} 31 \mathrm{HCC} 34 \mathrm{DI} 3$

H 3 CH4 2 HCC4 6 DI 4

P 3 CH5 2 HCC5 7 DI5

F 8 PF 3 FPC 2 DI6

H 8 PH 3 HPC 9 DI7

$\begin{array}{ll}\mathrm{CC} & 1.3664 \\ \mathrm{CC} 1 & 1.4335 \\ \mathrm{CH} 1 & 1.0846 \\ \mathrm{CH} 2 & 1.0827 \\ \mathrm{CH} 3 & 1.0923 \\ \mathrm{CH} 4 & 1.0884 \\ \mathrm{CH} 5 & 1.722 \\ \mathrm{PF} & 1.7812 \\ \mathrm{PH} & 1.416 \\ \mathrm{CCC} & 128.6095 \\ \mathrm{HCC} 1 & 121.4764 \\ \mathrm{HCC} 2 & 121.2028 \\ \mathrm{HCC} 3 & 116.3916 \\ \mathrm{HCC} 4 & 117.4576\end{array}$

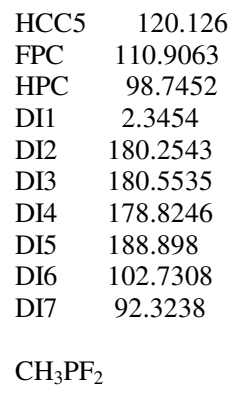

B3LYP

$\mathrm{C}$

$\mathrm{H} 1 \mathrm{CH} 1$

$\mathrm{H} 1 \mathrm{CH} 22 \mathrm{HCH}$

H 1 CH3 2 HCH2 3 DI1

P 1 PC 2 PCH 3 DI2

F 5 PF 1 FPC 2 DI4

F 5 PH 1 HPC 6 DI5

$\begin{array}{lc}\text { CH1 } & 1.0951 \\ \text { CH2 } & 1.0942 \\ \text { CH3 } & 1.0951 \\ \text { PC } & 1.8379 \\ \text { PF } & 1.6354 \\ \text { PH } & 1.6354 \\ \text { HCH } & 109.6859 \\ \text { HCH2 } & 108.5448 \\ \text { PCH } & 109.1578 \\ \text { FPC } & 97.3967 \\ \text { HPC } & 97.3939 \\ \text { DI1 } & 119.7912 \\ \text { DI2 } & -121.3568 \\ \text { DI4 } & 71.0816 \\ \text { DI5 } & 99.1918\end{array}$

$M P 2$

$\mathrm{C}$

$\mathrm{H} 1 \mathrm{CH} 1$

$\mathrm{H} 1 \mathrm{CH} 22 \mathrm{HCH}$

H 1 CH3 2 HCH2 3 DI1

P 1 PC 2 PCH 3 DI2

F 5 PF 1 FPC 2 DI4

F 5 PH 1 HPC 6 DI5

$\begin{array}{ll}\mathrm{CH} 1 & 1.09 \\ \mathrm{CH} 2 & 1.089 \\ \mathrm{CH} 3 & 1.09 \\ \mathrm{PC} & 1.8202 \\ \mathrm{PF} & 1.6349 \\ \mathrm{PH} & 1.6349 \\ \mathrm{HCH} & 109.6959 \\ \mathrm{HCH} 2 & 108.6365 \\ \mathrm{PCH} & 109.2652 \\ \mathrm{FPC} & 97.1982 \\ \mathrm{HPC} & 97.2 \\ \text { DI1 } & 119.9093 \\ \text { DI2 } & -120.9655 \\ \text { DI4 } & 70.9441 \\ \text { DI5 } & 99.2579\end{array}$

$\mathrm{CH}_{2} \mathrm{PF}_{2}^{-}$

\begin{tabular}{|c|c|}
\hline$B 3 L Y$ & \\
\hline $\mathrm{C}$ & \\
\hline$X 1 C$ & \\
\hline $\mathrm{H} 1 \mathrm{C}$ & $2 \mathrm{HCH}$ \\
\hline $\mathrm{H} 1 \mathrm{C}$ & $2 \mathrm{HCH} 23 \mathrm{DI} 1$ \\
\hline $\mathrm{P} 1 \mathrm{P}$ & PCH 3 DI2 \\
\hline F $5 \mathrm{P}$ & FPC 2 DI 4 \\
\hline F $5 \mathrm{P}$ & HPC 6 DI5 \\
\hline $\mathrm{CH} 1$ & 1.2194 \\
\hline $\mathrm{CH} 2$ & 1.0895 \\
\hline $\mathrm{CH} 3$ & 1.0879 \\
\hline
\end{tabular}




\begin{tabular}{|c|c|}
\hline $\mathrm{PC}$ & 1.6886 \\
\hline PF & 1.7268 \\
\hline $\mathrm{PH}$ & 1.7269 \\
\hline $\mathrm{HCH}$ & 91.2002 \\
\hline $\mathrm{HCH} 2$ & 90.4319 \\
\hline $\mathrm{PCH}$ & 88.3741 \\
\hline FPC & 106.2921 \\
\hline HPC & 106.292 \\
\hline DI1 & 117.9032 \\
\hline DI2 & -126.4534 \\
\hline DI4 & 44.5787 \\
\hline DI5 & 91.4448 \\
\hline \multicolumn{2}{|l|}{$M P 2$} \\
\hline \multicolumn{2}{|l|}{$\mathrm{C}$} \\
\hline \multicolumn{2}{|c|}{ X $1 \mathrm{CH} 1$} \\
\hline \multicolumn{2}{|c|}{$\mathrm{H} 1 \mathrm{CH} 22 \mathrm{HCH}$} \\
\hline \multicolumn{2}{|c|}{ H 1 CH3 2 HCH2 3 DI1 } \\
\hline \multicolumn{2}{|c|}{ P 1 PC 2 PCH 3 DI 2} \\
\hline \multirow{2}{*}{\multicolumn{2}{|c|}{ F 5 PF 1 FPC 2 DI 4}} \\
\hline & \\
\hline $\mathrm{CH} 1$ & 1.2194 \\
\hline $\mathrm{CH} 2$ & 1.0844 \\
\hline $\mathrm{CH} 3$ & 1.0831 \\
\hline $\mathrm{PC}$ & 1.6828 \\
\hline PF & 1.7246 \\
\hline $\mathrm{PH}$ & 1.7246 \\
\hline $\mathrm{HCH}$ & 94.4104 \\
\hline $\mathrm{HCH} 2$ & 85.404 \\
\hline $\mathrm{PCH}$ & 89.6862 \\
\hline FPC & 106.0799 \\
\hline HPC & 106.0855 \\
\hline DI1 & 118.2262 \\
\hline DI2 & -126.2888 \\
\hline DI4 & 49.6819 \\
\hline DI5 & 91.1323 \\
\hline \multicolumn{2}{|c|}{$B 3 L Y P /(D E L)$} \\
\hline \multicolumn{2}{|c|}{ C } \\
\hline \multicolumn{2}{|c|}{$\mathrm{H} 1 \mathrm{CH} 1$} \\
\hline \multicolumn{2}{|c|}{$\mathrm{H} 1 \mathrm{CH} 22 \mathrm{HCH}$} \\
\hline \multicolumn{2}{|c|}{ P 1 PC 2 PCH 3 DI 2} \\
\hline \multicolumn{2}{|c|}{ F 4 PF 1 FPC 2 DI 4} \\
\hline \multicolumn{2}{|c|}{ F 4 PH 1 HPC 5 DI5 } \\
\hline $\mathrm{CH} 1$ & 1.1091 \\
\hline $\mathrm{CH} 2$ & 1.1092 \\
\hline $\mathrm{PC}$ & 1.8145 \\
\hline $\mathrm{PF}$ & 1.6488 \\
\hline $\mathrm{PH}$ & 1.6491 \\
\hline $\mathrm{HCH}$ & 105.8836 \\
\hline $\mathrm{PCH}$ & 105.4918 \\
\hline FPC & 98.4003 \\
\hline HPC & 98.3942 \\
\hline DI2 & -248.4737 \\
\hline DI4 & 74.6 \\
\hline DI5 & 99.0935 \\
\hline
\end{tabular}

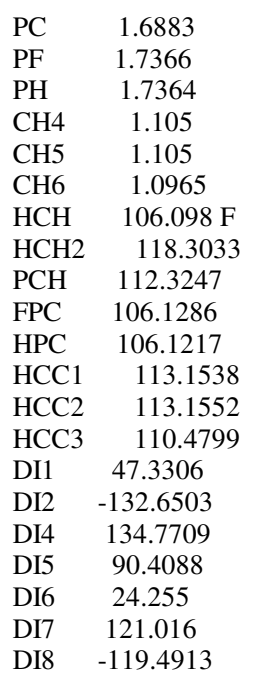

$M P 2$

$\mathrm{C}$

$\mathrm{H} 1 \mathrm{CH} 1$

$\mathrm{X} 1 \mathrm{CH} 22 \mathrm{HCH}$

C 1 CH3 2 HCH2 3 DI 1

P 1 PC 2 PCH 3 DI2

F 5 PF 1 FPC 2 DI4

F 5 PH 1 HPC 6 DI5

H 4 CH4 1 HCC1 3 DI6

$\mathrm{H} 4 \mathrm{CH} 51$ HCC2 8 DI7

H 4 CH6 1 HCC3 8 DI8

$\begin{array}{lc}\text { CH1 } & 1.0869 \\ \text { CH2 } & 1.0992 \\ \text { CH3 } & 1.5066 \\ \text { PC } & 1.6839 \\ \text { PF } & 1.7346 \\ \text { PH } & 1.7345 \\ \text { CH4 } & 1.0975 \\ \text { CH5 } & 1.0975 \\ \text { CH6 } & 1.0919 \\ \text { HCH } & 106.098 \\ \text { HCH2 } & 119.1317 \\ \text { PCH } & 112.6525 \\ \text { FPC } & 105.5894 \\ \text { HPC } & 105.586 \\ \text { HCC1 } & 112.8985 \\ \text { HCC2 } & 112.8996 \\ \text { HCC3 } & 109.8978 \\ \text { DI1 } & 43.2474 \\ \text { DI2 } & -136.7293 \\ \text { DI4 } & 135.047 \\ \text { DI5 } & 89.8754 \\ \text { DI6 } & 20.8078 \\ \text { DI7 } & 121.3994 \\ \text { DI8 } & -119.3001 \\ & \end{array}$

$\mathrm{C}_{3} \mathrm{H}_{4} \mathrm{PF}_{2}^{-}$

$\mathrm{C}_{2} \mathrm{H}_{4} \mathrm{PF}_{2}^{-}$

B3LYP

$\mathrm{C}$

$\mathrm{H} 1 \mathrm{CH} 1$

$\mathrm{X} 1 \mathrm{CH} 22 \mathrm{HCH}$

C 1 CH3 2 HCH2 3 DI 1

P 1 PC 2 PCH 3 DI2

F 5 PF 1 FPC 2 DI4

F 5 PH 1 HPC 6 DI5

H 4 CH4 1 HCC1 3 DI6

$\mathrm{H} 4 \mathrm{CH} 51$ HCC2 8 DI7

H 4 CH6 1 HCC3 8 DI8

\begin{tabular}{|c|c|}
\hline $\mathrm{CH} 2$ & 1.0868 \\
\hline $\mathrm{CH} 3$ & 1.0958 \\
\hline $\mathrm{CH} 4$ & 1.0916 \\
\hline CH5 & 1.7138 \\
\hline $\mathrm{PF}$ & 1.7063 \\
\hline $\mathrm{PH}$ & 1.7064 \\
\hline $\mathrm{CCC}$ & 129.1637 \\
\hline $\mathrm{HCC} 1$ & 121.7445 \\
\hline $\mathrm{HCC} 2$ & 121.1624 \\
\hline $\mathrm{HCC} 3$ & 115.8707 \\
\hline $\mathrm{HCC} 4$ & 119.1384 \\
\hline HCC5 & 119.8439 \\
\hline FPC & 104.5487 \\
\hline HPC & 104.5576 \\
\hline DI1 & 0.0057 \\
\hline DI2 & 179.9913 \\
\hline DI3 & 180.0033 \\
\hline DI4 & 180.0002 \\
\hline DI5 & 180.0095 \\
\hline DI6 & 134.0116 \\
\hline DI7 & 92.015 \\
\hline \multicolumn{2}{|l|}{$M P 2$} \\
\hline \multicolumn{2}{|l|}{$\mathrm{C}$} \\
\hline \multicolumn{2}{|c|}{ C $1 \mathrm{CC}$} \\
\hline \multicolumn{2}{|c|}{ C 2 CC $11 \mathrm{CCC}$} \\
\hline \multicolumn{2}{|c|}{ H 1 CH1 2 HCC 13 DI 1} \\
\hline \multicolumn{2}{|c|}{$\mathrm{H} 1 \mathrm{CH} 22 \mathrm{HCC} 24 \mathrm{DI} 2$} \\
\hline \multicolumn{2}{|c|}{$\mathrm{H} 2 \mathrm{CH} 31 \mathrm{HCC} 34 \mathrm{DI} 3$} \\
\hline \multicolumn{2}{|c|}{$\mathrm{H} 3 \mathrm{CH} 42 \mathrm{HCC} 46$ DI 4} \\
\hline \multicolumn{2}{|c|}{ P 3 CH5 2 HCC5 7 DI5 } \\
\hline \multicolumn{2}{|c|}{ F 8 PF 3 FPC 2 DI6 } \\
\hline & II \\
\hline
\end{tabular}

$\begin{array}{ll}\text { CC } & 1.3681 \\ \text { CC1 } & 1.4285 \\ \text { CH1 } & 1.0887 \\ \text { CH2 } & 1.0868 \\ \text { CH3 } & 1.0958 \\ \text { CH4 } & 1.0916 \\ \text { CH5 } & 1.7138 \\ \text { PF } & 1.7063 \\ \text { PH } & 1.7064 \\ \text { CCC } & 129.1637 \\ \text { HCC1 } & 121.7445 \\ \text { HCC2 } & 121.1624 \\ \text { HCC3 } & 115.8707 \\ \text { HCC4 } & 119.1384 \\ \text { HCC5 } & 119.8439 \\ \text { FPC } & 104.5487 \\ \text { HPC } & 104.5576 \\ \text { DI1 } & 0.0057 \\ \text { DI2 } & 179.9913 \\ \text { DI3 } & 180.0033 \\ \text { DI4 } & 180.0002 \\ \text { DI5 } & 180.0095 \\ \text { DI6 } & 134.0116 \\ \text { DI7 } & 92.015\end{array}$

$\mathrm{CH}_{3} \mathrm{POF}_{2}$

\begin{tabular}{|c|c|c|c|}
\hline \multicolumn{4}{|c|}{$B 3 L Y P$} \\
\hline \multicolumn{2}{|c|}{$\mathrm{C}$} & \multicolumn{2}{|c|}{$B 3 L Y P$} \\
\hline \multicolumn{2}{|c|}{$\mathrm{C} 1 \mathrm{CC}$} & \multicolumn{2}{|l|}{$\mathrm{C}$} \\
\hline \multicolumn{2}{|c|}{$\mathrm{C} 2 \mathrm{CC} 11 \mathrm{CCC}$} & \multicolumn{2}{|c|}{ H 1 CH1 } \\
\hline \multicolumn{2}{|c|}{ H 1 CH1 2 HCC 13 DI 1} & \multicolumn{2}{|c|}{$\mathrm{H} 1 \mathrm{CH} 22 \mathrm{HCH}$} \\
\hline \multicolumn{2}{|c|}{$\mathrm{H} 1 \mathrm{CH} 22 \mathrm{HCC} 24 \mathrm{DI} 2$} & \multicolumn{2}{|c|}{$\mathrm{H} 1 \mathrm{CH} 32 \mathrm{HCH} 23 \mathrm{DI} 1$} \\
\hline \multicolumn{2}{|c|}{$\mathrm{H} 2 \mathrm{CH} 31 \mathrm{HCC} 34 \mathrm{DI} 3$} & \multicolumn{2}{|c|}{ P 1 PC 2 PCH 3 DI 2} \\
\hline \multicolumn{2}{|c|}{ H 3 CH4 2 HCC4 6 DI 4} & \multicolumn{2}{|c|}{ F 5 PF 1 FPC 2 DI 4} \\
\hline \multicolumn{2}{|c|}{ P 3 CH5 2 HCC5 7 DI5 } & \multicolumn{2}{|c|}{ F 5 PH 1 HPC 6 DI5 } \\
\hline \multicolumn{2}{|c|}{ F 8 PF 3 FPC 2 DI6 } & \multicolumn{2}{|c|}{ O 5 PO 1 OPC 6 DI6 } \\
\hline \multicolumn{4}{|c|}{ F 8 PH 3 HPC 9 DI7 } \\
\hline & & $\mathrm{CH} 1$ & 1.0926 \\
\hline & & $\mathrm{CH} 2$ & 1.092 \\
\hline & 1.3681 & $\mathrm{CH} 3$ & 1.0927 \\
\hline $\mathrm{CC} 1$ & 1.4285 & $\mathrm{PC}$ & 1.7982 \\
\hline $\mathrm{CH} 1$ & 1.0887 & PF & 1.5927 \\
\hline
\end{tabular}




\begin{tabular}{|c|c|}
\hline $\mathrm{PH}$ & 1.593 \\
\hline $\mathrm{PO}$ & 1.4693 \\
\hline $\mathrm{HCH}$ & 109.9756 \\
\hline $\mathrm{HCH} 2$ & 109.0214 \\
\hline $\mathrm{PCH}$ & 108.7891 \\
\hline FPC & 103.4955 \\
\hline HPC & 103.42 \\
\hline OPC & 119.1096 \\
\hline DI1 & 120.5295 \\
\hline DI2 & -121.1637 \\
\hline DI4 & 69.5596 \\
\hline DI5 & 102.2135 \\
\hline DI6 & -128.9842 \\
\hline \multicolumn{2}{|l|}{$M P 2$} \\
\hline \multicolumn{2}{|l|}{$\mathrm{C}$} \\
\hline \multicolumn{2}{|c|}{ H 1 CH1 } \\
\hline \multicolumn{2}{|c|}{$\mathrm{H} 1 \mathrm{CH} 22 \mathrm{HCH}$} \\
\hline \multirow{2}{*}{\multicolumn{2}{|c|}{$\begin{array}{l}\text { H } 1 \text { CH } 32 \text { HCH2 } 3 \text { DI1 } \\
\text { P } 1 \text { PC } 2 \text { PCH } 3 \text { DI } 2\end{array}$}} \\
\hline & \\
\hline \multicolumn{2}{|c|}{ F 5 PF 1 FPC 2 DI 4} \\
\hline \multicolumn{2}{|c|}{ F 5 PH 1 HPC 6 DI5 } \\
\hline \multicolumn{2}{|c|}{ O 5 PO 1 OPC 6 DI6 } \\
\hline $\mathrm{CH} 1$ & 1.0876 \\
\hline $\mathrm{CH} 2$ & 1.0871 \\
\hline $\mathrm{CH} 3$ & 1.0876 \\
\hline $\mathrm{PC}$ & 1.7872 \\
\hline $\mathrm{PF}$ & 1.5932 \\
\hline $\mathrm{PH}$ & 1.5933 \\
\hline $\mathrm{PO}$ & 1.4745 \\
\hline $\mathrm{HCH}$ & 109.9592 \\
\hline $\mathrm{HCH} 2$ & 109.1825 \\
\hline $\mathrm{PCH}$ & 108.7945 \\
\hline FPC & 103.1756 \\
\hline HPC & 103.1583 \\
\hline $\mathrm{OPC}$ & 119.0719 \\
\hline DI1 & 120.7141 \\
\hline DI2 & -120.7143 \\
\hline DI4 & 69.4855 \\
\hline DI5 & 102.0274 \\
\hline DI6 & -128.9961 \\
\hline \multicolumn{2}{|c|}{$\mathrm{CH}_{2} \mathrm{POF}_{2}^{-}$} \\
\hline \multicolumn{2}{|c|}{$B 3 L Y P$} \\
\hline \multicolumn{2}{|c|}{$\mathrm{C}$} \\
\hline \multicolumn{2}{|c|}{$\mathrm{H} 1 \mathrm{CH} 1$} \\
\hline \multicolumn{2}{|c|}{$\mathrm{H} 1 \mathrm{CH} 22 \mathrm{HCH}$} \\
\hline $\mathrm{X} 1 \mathrm{Cl}$ & I3 2 HCH2 3 DI1 \\
\hline P 1 PC & 2 PCH 3 DI2 \\
\hline F $5 \mathrm{PF}$ & 1 FPC 2 DI4 \\
\hline F $5 \mathrm{PH}$ & 1 HPC 6 DI5 \\
\hline $\mathrm{O} 5 \mathrm{PC}$ & 1 OPC 6 DI6 \\
\hline $\mathrm{CH} 1$ & 1.0828 \\
\hline $\mathrm{CH} 2$ & 1.0842 \\
\hline $\mathrm{CH} 3$ & 1.0927 \\
\hline $\mathrm{PC}$ & 1.6721 \\
\hline $\mathrm{PF}$ & 1.664 \\
\hline $\mathrm{PH}$ & 1.648 \\
\hline PO & 1.4976 \\
\hline $\mathrm{HCH}$ & 119.4329 \\
\hline $\mathrm{HCH} 2$ & 109.0214 \\
\hline $\mathrm{PCH}$ & 117.1751 \\
\hline FPC & 112.7351 \\
\hline HPC & 108.2082 \\
\hline $\mathrm{OPC}$ & 122.5852 \\
\hline DI1 & 120.5295 \\
\hline DI2 & -161.3376 \\
\hline DI4 & 113.7594 \\
\hline DI5 & 98.3163 \\
\hline DI6 & -131.4692 \\
\hline$M P 2$ & \\
\hline $\begin{array}{l}\mathrm{C} \\
\mathrm{H} 1 \mathrm{C}\end{array}$ & \\
\hline
\end{tabular}

$\mathrm{H} 1 \mathrm{CH} 22 \mathrm{HCH}$

$\mathrm{X} 1 \mathrm{CH} 32 \mathrm{HCH} 23 \mathrm{DI} 1$

P 1 PC 2 PCH 3 DI2

F 5 PF 1 FPC 2 DI4

F 5 PH 1 HPC 6 DI5

O 5 PO 1 OPC 6 DI6

$\begin{array}{lc}\text { CH1 } & 1.0798 \\ \text { CH2 } & 1.081 \\ \text { CH3 } & 1.0927 \\ \text { PC } & 1.6717 \\ \text { PF } & 1.6657 \\ \text { PH } & 1.6431 \\ \text { PO } & 1.5017 \\ \text { HCH } & 118.4282 \\ \text { HCH2 } & 109.0214 \\ \text { PCH } & 116.0333 \\ \text { FPC } & 113.6518 \\ \text { HPC } & 106.8916 \\ \text { OPC } & 122.5 \\ \text { DI1 } & 120.5295 \\ \text { DI2 } & -151.0441 \\ \text { DI4 } & 104.8188 \\ \text { DI5 } & 98.3796 \\ \text { DI6 } & -131.8326\end{array}$

$B 3 L Y P /(D E L)$

$\mathrm{C}$

$\mathrm{H} 1 \mathrm{CH} 1$

$\mathrm{H} 1 \mathrm{CH} 22 \mathrm{HCH}$ P 1 PC 2 PCH 3 DI2

F 4 PF 1 FPC 2 DI4

F 4 PH 1 HPC 5 DI5

O 4 PO 1 OPC 5 DI6

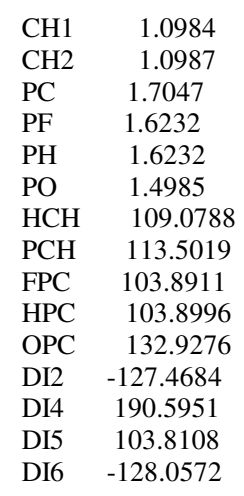

$\mathrm{C}_{2} \mathrm{H}_{4} \mathrm{POF}_{2}^{-}$

B3LYP

$\mathrm{X} 1 \mathrm{CH} 1$

$\mathrm{H} 1 \mathrm{CH} 22 \mathrm{HCH}$

C 1 CH3 2 HCH2 3 DI

P 1 PC 2 PCH 3 DI2

F 5 PF 1 FPC 2 DI4

F 5 PH 1 HPC 6 DI5

O 5 PO 1 OPC 6 DI6

H 4 CH4 1 HCC1 3 DI7

$\mathrm{H} 4 \mathrm{CH} 51$ HCC2 9 DI8

H 4 CH6 1 HCC3 9 DI9

$\begin{array}{ll}\mathrm{CH} 1 & 1.096 \\ \mathrm{CH} 2 & 1.0856 \\ \mathrm{CH} 3 & 1.5102 \\ \mathrm{PC} & 1.6699 \\ \mathrm{PF} & 1.668 \\ \mathrm{PH} & 1.6532 \\ \mathrm{PO} & 1.4981 \\ \mathrm{CH} 4 & 1.1032 \\ \mathrm{CH} 5 & 1.1073 \\ \mathrm{CH} 6 & 1.0977 \\ \mathrm{HCH} & 114.8319\end{array}$

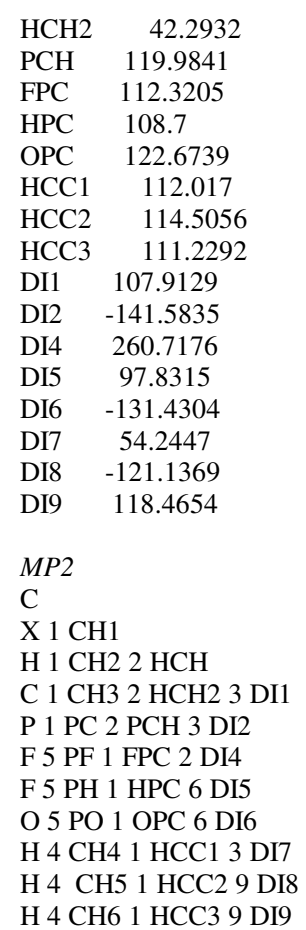

$\begin{array}{lc}\text { CH1 } & 1.096 \\ \text { CH2 } & 1.0821 \\ \text { CH3 } & 1.5082 \\ \text { PC } & 1.6679 \\ \text { PF } & 1.6699 \\ \text { PH } & 1.6491 \\ \text { PO } & 1.5025 \\ \text { CH4 } & 1.0959 \\ \text { CH5 } & 1.0998 \\ \text { CH6 } & 1.093 \\ \text { HCH } & 97.9362 \\ \text { HCH2 } & 46.4489 \\ \text { PCH } & 140.0013 \\ \text { FPC } & 112.8123 \\ \text { HPC } & 107.2193 \\ \text { OPC } & 123.0893 \\ \text { HCC1 } & 111.339 \\ \text { HCC2 } & 114.6189 \\ \text { HCC3 } & 110.7672 \\ \text { DI1 } & 123.8921 \\ \text { DI2 } & -143.7944 \\ \text { DI4 } & 251.6956 \\ \text { DI5 } & 97.5285 \\ \text { DI6 } & -131.909 \\ \text { DI7 } & 54.9399 \\ \text { DI8 } & -121.309 \\ \text { DI9 } & 118.0093 \\ & \end{array}$

$\mathrm{C}_{3} \mathrm{H}_{4} \mathrm{POF}_{2}^{-}$

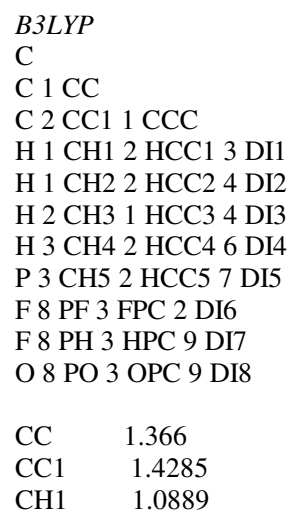




$\begin{array}{ll}\text { CH2 } & 1.0863 \\ \text { CH3 } & 1.0919 \\ \text { CH4 } & 1.0877 \\ \text { CH5 } & 1.6919 \\ \text { PF } & 1.6424 \\ \text { PH } & 1.6423 \\ \text { PO } & 1.4918 \\ \text { CCC } & 128.4336 \\ \text { HCC1 } & 121.9935 \\ \text { HCC2 } & 120.903 \\ \text { HCC3 } & 116.6516 \\ \text { HCC4 } & 120.5156 \\ \text { HCC5 } & 122.6195 \\ \text { FPC } & 108.9887 \\ \text { HPC } & 108.9769 \\ \text { OPC } & 122.5092 \\ \text { DI1 } & 0.0202 \\ \text { DI2 } & 179.9681 \\ \text { DI3 } & 180.0108 \\ \text { DI4 } & 179.9716 \\ \text { DI5 } & 180.0587 \\ \text { DI6 } & 130.7992 \\ \text { DI7 } & 98.3117\end{array}$

\begin{tabular}{|c|c|}
\hline DI8 & -130.8457 \\
\hline \multicolumn{2}{|l|}{$M P 2$} \\
\hline \multicolumn{2}{|l|}{$\mathrm{C}$} \\
\hline \multicolumn{2}{|c|}{$\mathrm{C} 1 \mathrm{CC}$} \\
\hline \multicolumn{2}{|c|}{$\mathrm{C} 2 \mathrm{CC} 11 \mathrm{CCC}$} \\
\hline \multicolumn{2}{|c|}{ H 1 CH1 2 HCC 13 DI 1} \\
\hline \multicolumn{2}{|c|}{$\mathrm{H} 1 \mathrm{CH} 22 \mathrm{HCC} 24$ DI 2} \\
\hline \multicolumn{2}{|c|}{$\mathrm{H} 2 \mathrm{CH} 31 \mathrm{HCC} 34 \mathrm{DI} 3$} \\
\hline \multicolumn{2}{|c|}{ H 3 CH4 2 HCC4 6 DI4 } \\
\hline \multicolumn{2}{|c|}{ P 3 CH5 2 HCC5 7 DI5 } \\
\hline \multicolumn{2}{|c|}{ F 8 PF 3 FPC 2 DI6 } \\
\hline \multirow{2}{*}{\multicolumn{2}{|c|}{$\begin{array}{l}\text { F } 8 \text { PH } 3 \text { HPC } 9 \text { DI7 } \\
\text { O } 8 \text { PO } 3 \text { OPC } 9 \text { DI8 }\end{array}$}} \\
\hline & \\
\hline $\mathrm{CC}$ & 1.3656 \\
\hline $\mathrm{CC} 1$ & 1.4292 \\
\hline $\mathrm{CH} 1$ & 1.0842 \\
\hline $\mathrm{CH} 2$ & 1.0818 \\
\hline $\mathrm{CH} 3$ & 1.0886 \\
\hline $\mathrm{CH} 4$ & 1.0836 \\
\hline CH5 & 1.6848 \\
\hline $\mathrm{PF}$ & 1.6419 \\
\hline
\end{tabular}

PH $\quad 1.6419$

PO 1.4972

CCC 127.9018

HCC1 121.7829

$\mathrm{HCC} 2 \quad 120.731$

HCC3 116.8746

HCC4 120.7983

HCC5 121.38

FPC 109.0855

HPC 109.0745

OPC $\quad 122.1148$

DI1 0.0078

DI2 179.9929

DI3 179.9983

DI4 179.9892

DI5 180.0447

DI6 130.7167

DI7 98.4789

DI8 $\quad-130.7618$ 